\title{
Optics-measurement-based beam position monitor calibrations in the LHC insertion regions
}

\author{
A. García-Tabarés Valdivieso $\odot^{*}$ and R. Tomás \\ CERN, 1211 Geneva 23, Switzerland
}

(Received 21 October 2019; accepted 31 March 2020; published 16 April 2020)

\begin{abstract}
Accurate measurements of optics functions, $\beta, \alpha, \mathrm{D}$, are essential for proper operation of a synchrotron both for machine protection and for performance. $\beta$ functions can be obtained from different observables: phase and amplitude of the transverse betatron oscillations and change of the tune by modulating the current of quadrupoles (K-modulation). Reconstruction of $\beta$ function using the betatron phase, in combination with K-modulation, has been the main measurement approach in the LHC IRs. Nonetheless, challenges have appeared in the $\beta$ calculation using these two techniques when aiming for smaller $\beta^{*}$, i.e., $\beta$-values at the interaction point (IP) in the LHC and the HL-LHC. The third $\beta$ measurement technique, based on the beam position monitor (BPM) signal amplitude, has not been used as widely in the LHC as the other methods since it requires accurate BPM gain calibration. This paper presents the development of a technique based on optics measurements to calibrate accurately LHC IR BPMs, allowing to use of the amplitude information in the measurement of IR $\beta$ functions.
\end{abstract}

DOI: $10.1103 /$ PhysRevAccelBeams.23.042801

\section{INTRODUCTION}

Linear optics measurements and corrections are key elements in the operation of present and future colliders such as LHC [1-4], its upgrades HL-LHC [5], HE-LHC [6], or the FCC $[7,8]$. The requirements of increasing the luminosity move the LHC into more challenging operational regimes with lower $\beta^{*}$.

In the LHC strong localized magnetic errors have to be corrected to achieve the design value of the $\beta$ function at the interaction point to provide the design luminosity within the 5\% tolerance limits to the experiments: ATLAS [9] and CMS [10]. Optics corrections are based on the analysis of turn-by-turn measurements from the beam position monitors (BPMs) [11-14] and K-modulation [15-18].

BPM calibration errors modify measured position by the calibration factor $C_{i}$, where $i$ is the BPM number. This is analytically described by:

$$
z_{i}^{\text {meas }}=C_{i} z_{i}^{\text {true }},
$$

where $z_{i}^{\text {true }}$ represents the true horizontal or vertical beam position at the $i$ th BPM and $z_{i}^{\text {meas }}$ is the reported measurement.

*ana.garcia-tabares.valdivieso@cern.ch

Published by the American Physical Society under the terms of the Creative Commons Attribution 4.0 International license. Further distribution of this work must maintain attribution to the author(s) and the published article's title, journal citation, and DOI.
At LHC, K-modulation allows to obtain the value of the average $\beta, \beta^{\mathrm{K} \text {-modulation }}$, at the quadrupoles placed closest to the IPs. The suitability of this method for future accelerators has been studied in HL-LHC, FCC, and SuperKEKB $[15-17,19]$. It has been found that the foreseen tune stability might not be sufficient for the good performance of this approach.

The optics measurements around the LHC use the turnby-turn frequency spectra obtained from BPM data. On the one hand, relative phase advances between a reference BPM and at least two other BPMs allow reconstructing the values of the $\beta$ functions at the reference BPM under the assumption that there are no optics errors between the concerned BPMs. This method, known as $\beta$ from phase $\left(\beta^{\phi}\right)$, was first used in LEP [20] and has been further developed in LHC, ALBA, and ESRF [21-24]. On the other hand, $\beta$ can be also computed directly from the amplitude of the turn-by-turn spectra. This approach is known as $\beta$ from amplitude $\left(\beta^{\mathrm{A}}\right)$. Nonetheless, the reconstruction of the $\beta$ function using the amplitude is biased by the calibration error of each BPM. The $\beta$ from amplitude approach has been used in the past [20,24-26] and it is currently implemented as part of the optics, measurements, and corrections (OMC) software [27]. However, this method has not been as widely used as $\beta^{\phi}$ or $\beta^{\mathrm{K} \text {-modulation }}$.

A precise knowledge of BPM calibration factors would allow us to accurately measure $\beta$ function using $\beta^{\mathrm{A}}$ approach where the performance of other approaches is limited. In low $\beta^{*}$ runs, the resolution required in the interaction region (IR) phase advance measurement is 
below the resolution that can be provided by the BPMs placed in the IR. In those scenarios, the amplitude of the transverse oscillations recorded at the BPMs closest to the IP increases as $\beta^{*}$ decreases, while the phase advance between neighbor BPMs is very small. $\beta^{\phi}$ approach is very sensitive to errors for values of the BPMs phase advance close to $n \pi$. To obtain the required $\beta$ accuracy, the BPM resolution should be ten times smaller than the best resolution achievable by the LHC BPMs [28]. Therefore, with the current resolution and reconstruction techniques, $\beta^{*}$ obtained using phase advance method in the IRs during the squeeze provides inaccurate values, which cannot be used for local corrections [29].

Turn-by-turn BPM data are also used for dispersion measurements, performed by shifting the frequency of the radio frequency (rf) systems in a range of $\pm 100 \mathrm{~Hz}$ for the LHC. Closed-orbit shift is measured using the zero-line frequency of the Fourier analysis. Dispersion function is then obtained by normalizing it by the momentum variation, $\Delta p / p$. BPM calibration factors bias as well dispersion calculation. In [30], normalized dispersion $D_{N, x}=\frac{D_{x}}{\sqrt{\beta_{x}^{A}}}$ is introduced as a calibration-independent observable since it is calculated as a ratio between two calibration-proportional quantities.

This paper introduces two optics-measurement-based BPM calibration measurement methods based on $\beta$-function and dispersion measurements. The two methods are based on measuring either the ratio $\sqrt{\beta^{\mathrm{A}} / \beta^{\phi}}$ or the ratio between horizontal normalized dispersion function times the $\sqrt{\beta_{x}^{\phi}}$ and the dispersion function $\left(D_{x} / D_{N, x} \sqrt{\beta_{x}^{\phi}}\right)$. Optics-measurement-based BPM calibration factors are calculated in an optics configuration where the lattice systematic errors affect as less as possible the $\beta^{\phi}$ and dispersion measurements. An optics that is suitable for the application of this method to the LHC IR BPMs is the ballistic or alignment optics, characterized by having the triplets switched off [31]. This optics configuration was first designed for alignment of the magnets placed in the triplet area, the Q1, Q2 and Q3 quadrupoles. An extended version of this optics, designed in 2017 specifically for the IRs BPM calibration studies, has Q4 quadrupoles also switched off and the dispersion in the IRs 1 and 5 not matched to zero [32]. A practical configuration of ballistic configuration is introduced in Sec. III.

Different types of BPMs are installed in LHC with different aims. They have been grouped according to the geometry of the pick-ups in the following categories: standard, enlarged aperture, and stripline as shown in Table I [33]. The largest number of BPMs are standard or cold button BPMs. Enlarged aperture button BPMs are placed close to the recombination dipoles. Stripline or directional BPMs, able to measure the beam direction, are placed in the common areas where both beams circulate in one vacuum pipe. The calibration analysis has been focused
TABLE I. Summary of BPM characteristics.

\begin{tabular}{|c|c|c|c|c|}
\hline Name & Stripline & Stripline & rged aperture & Standard \\
\hline Geometry & Stripline & Stripline & Button & Button \\
\hline LHC name & BPMS & BPMSX & BPMW & BPM \\
\hline Diameter & $61 \mathrm{~mm}$ & $81 \mathrm{~mm}$ & $61 \mathrm{~mm}$ & $49 \mathrm{~mm}$ \\
\hline
\end{tabular}

on the IRs BPMs, since during the annual LHC commissioning $[34,35]$ a systematic difference between the results obtained using $\beta^{\phi}$ and $\beta^{\mathrm{A}}$ was observed in these regions as shown in Fig. 1, where the horizontal (top) and vertical (bottom) deviations are shown as function of the BPM type. The average $\beta$-beating, $\left\langle\left(\beta^{\mathrm{A}}-\beta^{\phi}\right) / \beta^{\phi}\right\rangle$ between the two techniques, illustrates that a systematic lower value is obtained in the $\beta^{\mathrm{A}}$ with respect to the $\beta^{\phi}$ in the case of stripline and enlarged aperture BPMs. The data have been acquired in four consecutive years by using the injection optics. The reason for this calibration errors remains unknown and it has to be addressed via measurements with beam.

The systematic difference between the two $\beta$ calculations is also evident in Fig. 2 where the combined histogram of the ratio $\sqrt{\beta^{\mathrm{A}} / \beta^{\phi}}$ measured for injection, flattop, ballistic and high- $\beta^{*}$ optics at the stripline BPMs is shown.

The paper is structured as follows. Section II summarizes the methods used to measure $\beta$ function and dispersion in the LHC, including their limitations and how to obtain BPM calibration factors. A description of the ballistic optics together with the calibration factors obtained using both $\beta$ function and dispersion are present in Sec. III.

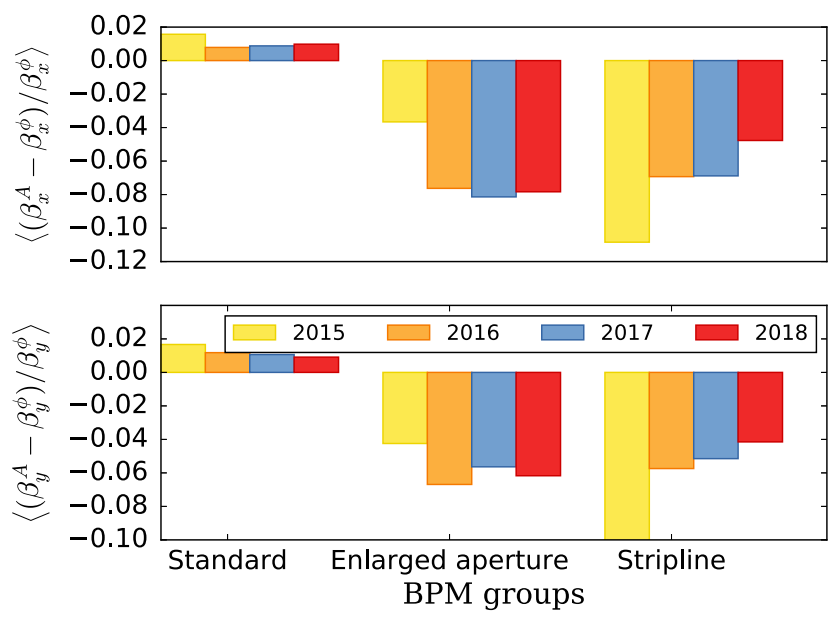

FIG. 1. Top: Average horizontal amplitude $\beta$-beating with respect to $\beta^{\phi}$, Bottom: Average vertical amplitude $\beta$-beating with respect to $\beta^{\phi}$. The data, acquired for four consecutive years, refer to the injection optics. The BPMs have been divided in groups depending on their geometry according to Table I. 

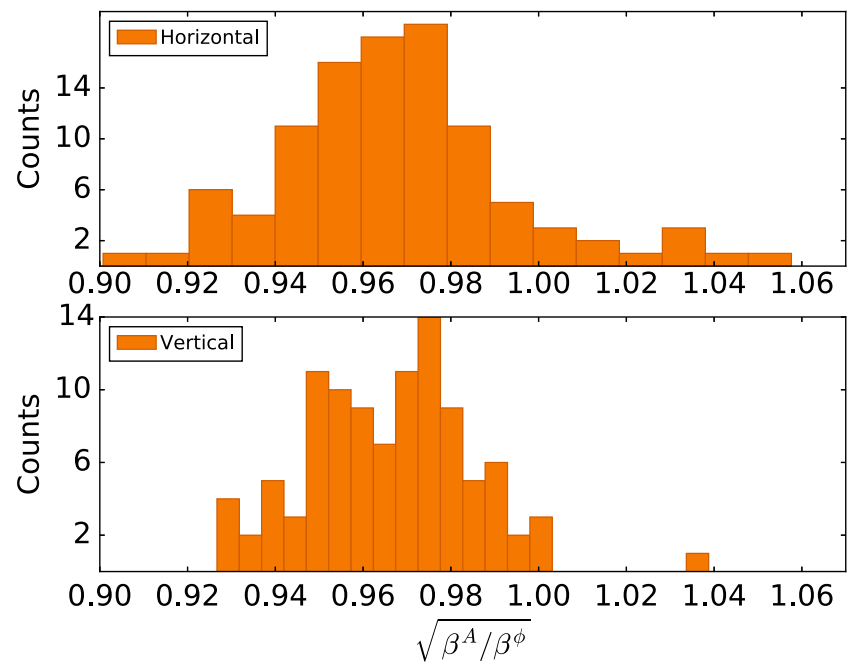

FIG. 2. Combined histogram of the ratio $\sqrt{\beta^{\mathrm{A}} / \beta^{\phi}}$ in the stripline BPMs measured in 2017 using different optics configurations: injection, flattop, high- $\beta^{*}$ and ballistic.

Section IV compares the $\beta$-function calculated for different optics configurations with and without recalibrating the BPMs and compares these results to the results obtained with the $\beta^{\phi}$ and the $\beta^{\mathrm{K} \text {-modulation }}$ approaches.

\section{OPTICS MEASUREMENTS IN LHC: METHODS AND LIMITATIONS}

\section{A. $\boldsymbol{\beta}$-function measurements based on K-modulation}

The most common method to measure $\beta^{*}$ in the LHC has been K-modulation $[15,17,18]$. K-modulation approach is based on the analysis of the tune change induced by a current modulation in the quadrupoles located left and right to the IP.

Accuracy of this method depends on the power supplies control and natural tune stability. Accuracy of the $\beta^{*}$ measurement for the LHC and high luminosity LHC using K-modulation has been previously studied in [15]. The study concludes that accuracy on the tune measurement of about $10^{-5}$ is crucial to control the $\beta^{*}$ within the tolerance constraints given by the luminosity. Analysis of the tune stability [36] performed during the second run of the LHC shows that the tune jitter ranges between $2 \times$ $10^{-5}$ and $10^{-4}$ depending on the optics configuration analyzed. Those fluctuations exceed the tolerances set by the LHC experiments.

\section{B. $\beta$-function measurements based on turn-by-turn data}

LHC turn-by-turn optics measurements are based on the analysis of AC-dipole driven oscillations which can be observed in the BPMs [37]. This data is post-processed using Fourier transformation methods to obtain the amplitude and phase of each spectral line [38]. These values are later analyzed for the reconstruction of the different optics parameters. Linear optics studies are specially focused on the analysis of amplitude and phase corresponding to the main line of the spectrum, associated to the driven tune. In this section we focus on the $\beta$ from amplitude technique, as its random and systematic errors are not addressed elsewhere, contrary to $\beta$ from phase [21-24]. For the $i$ th BPM, true amplitude and phase are related to the beam position, through:

$$
z_{i}^{\mathrm{D}}(n)=A_{z, i}^{\mathrm{D}} \sin \left(\mu_{z, i}^{\mathrm{D}}+2 \pi Q_{z}^{\mathrm{D}} n\right),
$$

where $A_{z, i}^{\mathrm{D}}, \mu_{z, i}^{\mathrm{D}}, Q_{z}^{D}$, and $n$ are the amplitude, the phase, the tune of the driven motion, and the turn number, respectively. The subscript $\mathrm{z}$ represents a generic plane, $\mathrm{x}$ or $\mathrm{y}$. The amplitude, $A_{z, i}^{\mathrm{D}}$, can also be expressed in terms of the driven $\beta$ function, $\beta_{z, i}^{\mathrm{D}}$, and a common observable for all BPMs, the driven action, $2 J_{z}^{\mathrm{D}}$,

$$
A_{z, i}^{\mathrm{D}}=\sqrt{2 J_{z}^{\mathrm{D}} \beta_{z, i}^{\mathrm{D}}} .
$$

To simplify the equations, the subindex $z$ is omitted in the following. Since measurement of the oscillation amplitude is biased by the individual BPM calibration factors, $\mathrm{C}_{i}$, measured amplitude, $\mathrm{A}_{i}^{\mathrm{D} \text {,meas }}$, deviates from Eq. (3) as:

$$
A_{i}^{\mathrm{D}, \text { meas }}=C_{i} \sqrt{2 J^{\mathrm{D}} \beta_{i}^{\mathrm{D}}} .
$$

The amplitude of the driven transverse excitations, $\mathrm{A}_{i}^{\mathrm{D} \text {,meas }}$, is obtained from the Fourier analysis of the turn-by-turn data, and is the basis for determining $\beta^{\mathrm{A}}$.

In order to obtain the value of the action induced by the ac-dipole, it is necessary to normalize the square of the amplitude of the transversal excitations, $\left(\mathrm{A}_{i}^{\mathrm{D} \text {,meas }}\right)^{2}$, by the $\beta_{i}^{\text {D }}$ function. This value can be obtained in two ways, using either the measured $\beta_{i}^{\phi, \mathrm{D}}$ or the model $\beta_{i}^{\text {model, } \mathrm{D}}$ given by MADX [39].

From Eq. (4) it is

$$
\frac{2 J^{\mathrm{D}}}{\mathrm{N}} \sum_{i=1}^{\mathrm{N}}\left(C_{i}\right)^{2}=\frac{1}{\mathrm{~N}} \sum_{i=1}^{\mathrm{N}} \frac{\left(A_{i}^{\mathrm{D}, \text { meas }}\right)^{2}}{\beta_{i}^{\mathrm{D}}},
$$

where $\mathrm{N}$ is the number of BPMs.

Since largest discrepancies between the $\beta^{\phi}$ and $\beta^{\mathrm{A}}$ occur for the stripline BPMs placed close to the IP, and in order to minimize the impact of the calibration factors, the summation is restricted to a subset of $\mathrm{N}^{\prime}$ BPMs placed in the arcs. Equation (5) can be rewritten as:

$$
\frac{2 J^{\mathrm{D}}}{\mathrm{N}^{\prime}} \sum_{i=1}^{\mathrm{N}^{\prime}}\left(C_{i}\right)^{2}=\frac{1}{\mathrm{~N}^{\prime}} \sum_{i=1}^{\mathrm{N}^{\prime}} \frac{\left(A_{i}^{\mathrm{D}, \text { meas }}\right)^{2}}{\beta_{i}^{\mathrm{D}}} .
$$

In order to simplify the notation, the product of the action times the average of the calibration factors square on the 
left hand size will be denoted as calibration-biased action, given by:

$$
2 J_{C}^{\mathrm{D}}=\overline{(C)^{2}} 2 J^{\mathrm{D}}=\frac{1}{\mathrm{~N}^{\prime}} \sum_{i=1}^{\mathrm{N}^{\prime}} \frac{\left(\mathrm{A}_{i}^{\mathrm{D}, \text { meas }}\right)^{2}}{\beta_{i}^{\mathrm{D}}} .
$$

Once the calibration biased action is calculated, the driven $\beta$-function at a given BPM, $\beta_{i}^{\mathrm{A}, \mathrm{D}}$, can be computed by normalizing the amplitude by the driven action

$$
\beta_{i}^{\mathrm{A}, \mathrm{D}}=\frac{\left(A_{i}^{\mathrm{D}, \text { meas }}\right)^{2}}{\overline{(C)^{2}} 2 J^{\mathrm{D}}}=\frac{\left(A_{i}^{\mathrm{D}, \text { meas }}\right)^{2}}{2 J_{C}^{\mathrm{D}}} .
$$

Using Eq. (2), Eq. (8) becomes:

$$
\beta_{i}^{\mathrm{A}, \mathrm{D}}=\frac{\left(C_{i}\right)^{2} \beta_{i}^{\mathrm{D}}}{\overline{(C)^{2}}}
$$

which shows that the $\beta_{i}^{\mathrm{A}, \mathrm{D}}$ calculation is affected by a factor $\left(C_{i}\right)^{2} / \overline{(C)^{2}}$, i.e, including both the average arc calibration factors and the individual calibration factor.

It is known [37] that the ac dipole introduces an optics perturbation. The relationship between the beta function with and without ac dipole is

$$
\beta_{i}^{\mathrm{A}}=\beta_{i}^{\mathrm{A}, \mathrm{D}} \frac{1+\lambda^{2}-2 \lambda \cos \left(2 \phi_{i \Rightarrow \text { ac-dipole }}-2 \pi Q\right)}{1-\lambda^{2}}
$$

where $\lambda$ is related to the separation between the natural tune $(Q)$ and driven tune $\left(Q^{\mathrm{D}}\right)$, by $\lambda=\frac{\sin \left[\pi\left(\mathrm{Q}^{\mathrm{D}}-\mathrm{Q}\right)\right]}{\left.\sin \left[\pi\left(\mathrm{Q}^{\mathrm{D}}+\mathrm{Q}\right)\right)\right]}$. The term $\phi_{i \Rightarrow \text { AC-dipole }}$ correspond to the phase advance between a given BPM and the ac-dipole. The following section studies the $\beta_{i}^{\mathrm{A}}$ uncertainty, $\Delta \beta_{i}^{\mathrm{A}}$.

\section{Action uncertainty analysis for $\beta^{\mathrm{A}}$ calculation}

The action, $2 J_{C}^{\mathrm{D}, \phi}$, can be calculated using a previously computed $\beta$ function from phase, $\beta_{i}^{\mathrm{D}, \phi}$ by averaging over the arc BPMs:

$$
2 J_{C}^{\mathrm{D}, \phi}=\frac{1}{\mathrm{~N}^{\prime}} \sum_{i=1}^{\mathrm{N}^{\prime}} \frac{\left(\mathrm{A}_{i}^{\mathrm{D}, \text { meas }}\right)^{2}}{\beta_{i}^{D, \phi}}=\frac{1}{\mathrm{~N}^{\prime}} \sum_{i=1}^{\mathrm{N}^{\prime}} \frac{\left(C_{i} \mathrm{~A}_{i}^{\mathrm{D}}\right)^{2}}{\beta_{i}^{D, \phi}} .
$$

Equation (11) can be simplified by denoting $2 J_{i}^{\mathrm{D}, \phi}=\frac{\left(A_{i}^{\mathrm{D}}\right)^{2}}{\beta_{i}^{\mathrm{D}, \phi}}$. In the past, $2 J_{C}^{\mathrm{D}}$ was computed using a model value established using MAD-X $-\beta_{i}^{D \text {,model }}$ - instead of $\beta_{i}^{D, \phi}$.

In the Appendix $A$ it is shown that the average of $\Delta \beta / \beta^{\text {model }}$ increases quadratically with the gradient errors [see Eq. (A2)]. Therefore, under the assumption that $\beta^{\phi}$ gives an accurate value of the actual $\beta^{\phi}$, it is appropriate using $\beta^{\phi}$ rather than $\beta^{\text {model }}$ for computing $2 J_{C}^{D}$.

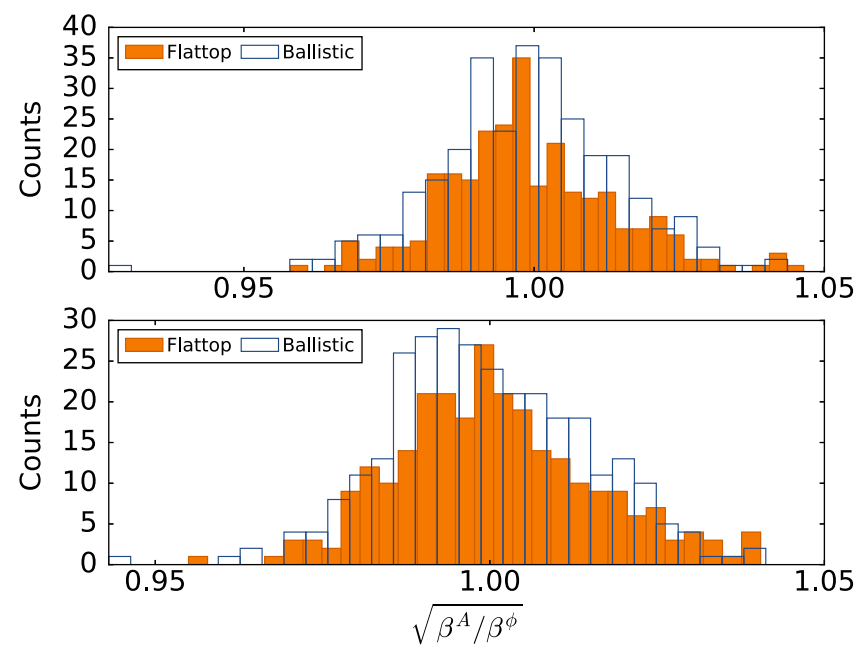

FIG. 3. Histograms of the ratio $\sqrt{\beta^{\mathrm{A}} / \beta^{\phi}}$ - top horizontal plane and bottom vertical plane-in the standard BPMs measured in different optics configurations in 2017, with a spread of about $1.5 \%$.

Calibration factors are a challenging limitation for the $\beta^{\mathrm{A}}$ method. This section focuses on the calibration factors associated to the button BPMs since the calibration biased action is calculated using only this kind of monitors. Figure 3 shows that calibration factors, estimated using the ratio $\sqrt{\beta^{\mathrm{A}} / \beta^{\phi}}$, follow in first approximation a Gaussian distribution with a spread $\sigma(C)$ of about $1.5 \%$.

The calibration factors $C_{i}$ can be splitted in two terms: an average term $\bar{C}$ and an individual spread term $\tilde{C}_{i}$ as $C_{i}=\bar{C}+\tilde{C}_{i}$. Thus the calibration biased action writes,

$$
2 J_{C}^{\mathrm{D}, \phi}=\frac{1}{\mathrm{~N}^{\prime}} \sum_{i=1}^{\mathrm{N}^{\prime}}\left(\bar{C}+\tilde{C}_{i}\right)^{2} 2 J_{i}^{\mathrm{D}, \phi}=\overline{2 J}_{C}^{\mathrm{D}, \phi}+\widetilde{2 J}_{C}^{\mathrm{D}, \phi}
$$

where:

$$
\begin{aligned}
\overline{2 J_{C}^{\mathrm{D}, \phi}}= & \frac{\bar{C}^{2}}{\mathrm{~N}^{\prime}} \sum_{i=1}^{\mathrm{N}^{\prime}} 2 J_{i}^{D}+\frac{1}{\mathrm{~N}^{\prime}} \sum_{i=1}^{\mathrm{N}^{\prime}} 2 J_{i}^{D} \tilde{C}_{i}^{2} \\
\approx & \frac{\bar{C}^{2}}{\mathrm{~N}^{\prime}} \sum_{i=1}^{\mathrm{N}^{\prime}} 2 J_{i}^{D}+\frac{\sigma^{2}(C)}{\mathrm{N}^{\prime}} \sum_{i=1}^{\mathrm{N}^{\prime}} 2 J_{i}^{D} \\
= & \left(\bar{C}^{2}+\sigma^{2}(C)\right) \frac{1}{\mathrm{~N}^{\prime}} \sum_{i=1}^{\mathrm{N}^{\prime}} 2 J_{i}^{D} \\
& \widetilde{2 J}_{C}^{\mathrm{D}, \phi}=\frac{2 \bar{C}}{\mathrm{~N}^{\prime}} \sum_{i=1}^{\mathrm{N}^{\prime}} 2 J_{i}^{D} \widetilde{C}_{i} .
\end{aligned}
$$

$\overline{2 J}_{C}^{\mathrm{D}, \phi}$ is affected by the square of the average calibration, $\bar{C}^{2}$ and by its associated spread squared, $\sigma^{2}(C)$. This second contribution can be neglected. Therefore: 


$$
\frac{\sigma\left(2 J_{\mathrm{C}}^{\mathrm{D}, \phi}\right)}{2 J^{\mathrm{D}, \phi}} \approx 2 \sigma(C)
$$

The action value is computed averaging over the $\mathrm{N}^{\prime}$ button BPMs and therefore its associated relative error bar is given by the standard error of the average:

$$
\frac{\sigma\left(2 J_{\mathrm{C}}^{\mathrm{D}, \phi}\right)}{2 J_{\mathrm{C}}^{\mathrm{D}, \phi}} \approx \frac{2 \sigma(C)}{\sqrt{\mathrm{N}^{\prime}-1}},
$$

with $\sqrt{\mathrm{N}^{\prime}-1} \approx 17$ and $\sigma(C) \approx 1.5 \%$, the action relative error bar will be approximately a $0.2 \%$.

\section{2. $\beta^{\mathrm{A}}$ uncertainties analysis}

Reconstruction of $\beta$ function using the amplitude of the transverse oscillations is affected by uncertainties on different parameters: natural tunes $\left(\mathrm{Q}_{x, y}\right)$, driven tunes $\left(Q_{x, y}^{\mathrm{D}}\right)$, phase advance between the ac-dipole and the $i$ th BPM, $\phi_{i \Rightarrow \mathrm{AC}}$, amplitude fluctuations between BPMs and the action calculation $2 J_{\mathrm{C}}^{\mathrm{D}, \phi}$.

The natural tune uncertainty is given by a combination of the BBQ [40] system accuracy and by its stability as a function of time. This stability has been analyzed for several different optics in [36] concluding that in average the natural shift observed during the measurements fluctuates in a range between $2 \times 10^{-5}$ and $10^{-4}$. The analytic calculation of the tune error propagation in the $\beta$ function can be found in Appendix B. In the ballistic optics case, where the fractional part of the tunes are $\mathrm{Q}_{x}=0.28$, $\mathrm{Q}_{x}^{\mathrm{D}}=0.268, \mathrm{Q}_{y}=0.31$, and $\mathrm{Q}_{y}^{\mathrm{D}}=0.325$, the relative $\beta$-uncertainty is given by:

$$
\begin{gathered}
\frac{\partial \beta_{x, i}^{\mathrm{A}}}{\partial Q_{x}} \approx \beta_{x, i}^{\mathrm{A}, \mathrm{D}} \times 9.4, \\
\frac{\partial \beta_{y, i}^{\mathrm{A}}}{\partial Q_{y}} \approx \beta_{y, i}^{\mathrm{A}, \mathrm{D}} \times 9.1
\end{gathered}
$$

Equations (17) and (18) allow to estimate the direct effect of the tune uncertainty in the $\beta^{\mathrm{A}}$-error $\left(\sigma_{\beta^{\mathrm{A}}}\right)$. It can be seen that the tune error $\sigma_{Q_{x, y}}$ error gets amplified approximately 9 times in the $\beta^{\mathrm{A}}$-calculation.

On the other hand, the phase advance and the amplitude uncertainty are estimated during the turn-by-turn measurement analysis, by using single-value-decomposition (SVD) technique. The error introduced by the $\phi$-advance and the amplitude error in the $\beta$-function calculation is approximately $0.05 \%$ for a peak to peak amplitude excitation of approximately $2 \mathrm{~mm}$ recorded during 6600 turns.

The error introduced by the action calculation $2 J_{\mathrm{C}}^{\mathrm{D}, \phi}$ will propagate to the $\beta^{\mathrm{A}}$ :

$$
\frac{1}{\beta_{i}^{\mathrm{A}}} \frac{\partial \beta_{i}^{\mathrm{A}}}{\partial 2 J_{\mathrm{C}}^{\mathrm{D}, \phi}}=\frac{\sigma\left(2 J_{\mathrm{C}}^{\mathrm{D}, \phi}\right)}{2 J_{\mathrm{C}}^{\mathrm{D}, \phi}} .
$$

The length of the external excitation limits the number of turns available for the Fourier analysis. Since the ac-dipole cannot produce longer excitations than 6600 turns, the amount of data is increased by exciting the beam several times, $\mathrm{N}^{\mathrm{acq}}$, and by combining the different acquisitions to obtain a single $\beta$-value per BPM. In case of the $\beta^{\mathrm{A}}$ method, the final $\beta_{i}^{\mathrm{A}}$ value at a given BPM is calculated as an average over the number of measurements acquired, $\mathrm{N}_{\mathrm{acq}}$ :

$$
\left\langle\beta_{i}^{\mathrm{A}}\right\rangle=\frac{1}{\mathrm{~N}_{\mathrm{acq}}} \sum_{j=1}^{\mathrm{N}_{\mathrm{acq}}} \beta_{i, j}^{\mathrm{A}} .
$$

In this case, the error introduced by the machine fluctuation is given by the standard deviation of the acquisitions $\beta_{i}^{\mathrm{A}}$

$$
\sigma\left(\beta_{i}^{\mathrm{A}}\right)=\frac{1}{\mathrm{~N}_{\mathrm{acq}}} \sqrt{\sum_{j=1}^{\mathrm{N}_{\mathrm{acq}}}\left(\beta_{i, j}^{\mathrm{A}}-\left\langle\beta_{i}^{\mathrm{A}}\right\rangle\right)^{2}} .
$$

The total $\beta^{\mathrm{A}}$-error, $\Delta \beta_{i}^{\mathrm{A}}$, will be therefore given by the combination of three sources of errors:

$\left(\Delta \beta_{i}^{A}\right)^{2}=\left(\frac{\partial \beta_{i}^{\mathrm{A}}}{\partial 2 J_{C}^{\mathrm{D}, \phi}} \sigma\left(2 J_{C}^{\mathrm{D}, \phi}\right)\right)^{2}+\left(\frac{\partial \beta_{i}^{\mathrm{A}}}{\partial Q} \sigma(Q)\right)^{2}+\sigma\left(\beta_{i}^{\mathrm{A}}\right)^{2}$

This error-bar fluctuates between a $0.25 \%$ and a $0.4 \%$ depending on the optics configuration implemented in the accelerator.

\section{Optics-based calibration factors}

Optics-based calibration factors can be computed as the ratio between $\beta$ from amplitude, $\beta_{i}^{\mathrm{A}}$ and $\beta$ from phase, $\beta_{i}^{\phi}$,

$$
C_{\beta, i}=\sqrt{\frac{\beta_{i}^{\mathrm{A}}}{\beta_{i}^{\phi}}}=\frac{C_{i}}{\sqrt{\overline{\left(C_{i, \mathrm{ARCS}}\right)^{2}}}} .
$$

\section{Dispersion measurements}

Another optics function that could be used for opticsbased-BPM-calibration is the dispersion. The dispersion is measured by taking the average orbit of the turn-by-turn data at each BPM position. Changing the rf frequency, results in a change of the closed orbit due to dispersion, $\Delta C O_{x, i}$ : 


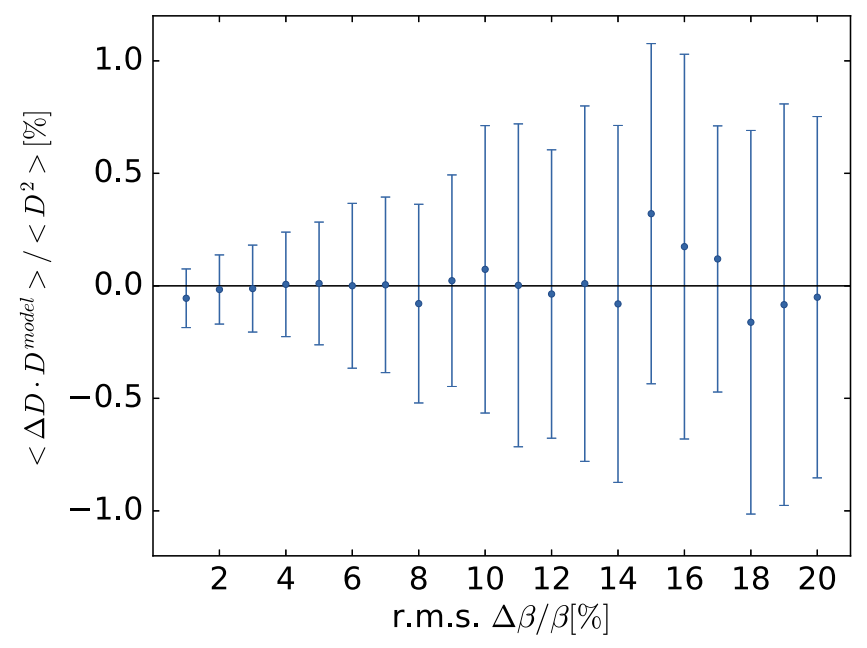

FIG. 4. Average dispersion-beating times dispersion model as a function of the rms $\beta$-beating.

$$
\Delta C O_{x, i}=\frac{\Delta p}{p} D_{x, i}
$$

Dispersion is then calculated at every BPM by a polynomial fit of the measured closed orbit over various momentum values. On the other hand, the momentum deviation $\frac{\Delta p}{p}$ is computed by using the dispersion predicted by the MADX model and the closed orbit which is affected by the calibration factor $C_{i}$ as:

$$
\frac{\Delta p}{p}^{\text {meas }}=\frac{\overline{D_{x, i}^{\text {model }} C_{i} \Delta C O_{x, i}}}{\left(\overline{\left.D_{x, i}^{\text {model }}\right)^{2}}\right.} .
$$

Closed orbit is the only direct measurement in Eq. (25). Fluctuations in this quantity can be analytically described as $\Delta C O_{x, i}=\Delta p / p\left(D^{\text {model }}+\Delta D\right)$, where $\Delta D$ represents variations the ideal model. Therefore the term, $D_{x, i}^{\text {model }} \Delta D$ evaluates the impact of the dispersion deviation with respect to the model in the momentum calculation. This calculation is based on the assumption that the average of the product of the dispersion predicted by the MADX model, $D_{x}^{\text {model }}$, times the dispersion-beating $\Delta D$ is 0 , independently of the magnetic errors presented in the machine. MADX simulations, including different quadrupolar errors, have been performed using ballistic configuration in order to validate this assumption, obtaining an average value that fluctuates around zero, as shown in Fig. 4.

Closed-orbit measurement is also affected by the individual BPM calibration factors, biasing the dispersion calculation:

$$
D_{x, i}^{\text {meas }}=\frac{C_{i} \Delta C O_{x, i}}{\Delta p / p^{\text {meas }}} \approx \frac{C_{i} \Delta C O_{x, i}}{\bar{C} \Delta p / p} .
$$

In [30] normalized dispersion is introduced as a calibration-independent observable, defined as the ratio:

$$
D_{N, x}=\frac{D_{x}}{\sqrt{\beta_{x}}}
$$

The normalized dispersion value can be computed using the weighted arithmetic mean of the ratio $C O_{x, i}^{\text {meas }} / A_{x, i}^{\text {meas }}$ over the number of acquisitions using the relative momentum deviation $\Delta p / p$ as individual weights.

\section{Dispersion-based calibration factors}

Dispersion-based calibration factors can be computed as the ratio between dispersion, $D_{x, i}^{\text {meas }}$, and normalized dispersion, $D_{N, x, i}^{\mathrm{meas}}$,

$$
C_{D, x, i}=\frac{D_{x, i}^{\text {meas }}}{D_{N, x, i}^{\text {meas }} \sqrt{\beta_{i}^{\phi}}}=\frac{C_{x, i}}{\overline{C_{x}}} .
$$

\section{BALLISTIC OPTICS}

Ballistic optics, the optics used for measuring the IR BPM calibration factors, defined by Eqs. (23) and (28) is described in this section. In this special optics configuration, magnets located in IR1 and IR5 common to both beams are switched off. This set of magnets, denoted as triplet are in charge of the beam final focusing adjustments. Switching off the focusing system presents some challenges for the machine operation that have to be taken into account. The main limitation comes from the large drift generated in the segment between the quadrupoles, leading to large values of the $\beta$ function in IR1 and IR5 for Beam 1 and 2 .

Additionally, a specific preparation is required before the start of the optics measurements. A process called degaussing is normally used to minimize the remanent magnetic field in the triplet. This procedure was applied to the triplet magnets at the beginning of the measurements to reduce the remaining field [41].

Figure 5 shows the quadrupolar strength of 2016 (top) and 2017 (bottom) ballistic optics. Magnets placed to the left of the dotted line in Fig. 5 are switched off, i.e., the triplet (Q1, Q2, and Q3) in 2016 and 2017 and additionally the Q4 only in 2017. By switching off the quadrupole Q4, the drift region is extended and so, the number of calibrated

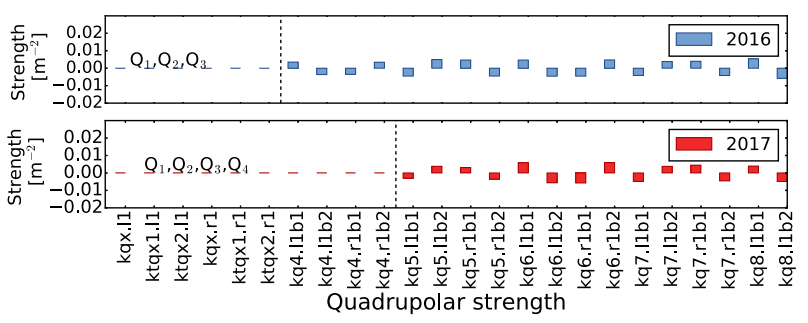

FIG. 5. Quadrupolar strength in the IR1 in 2016 (top) and in 2017 (bottom). The IR5 is equivalent. 

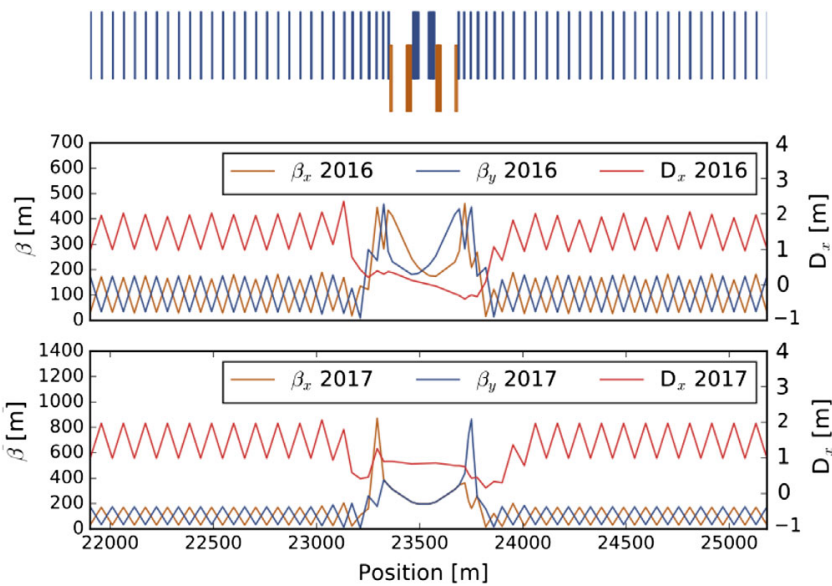

FIG. 6. Comparison of the horizontal model $\beta$, vertical model $\beta$ function and dispersion for the IR5: top 2016 and bottom 2017 (Beam 1).

BPMs. The extra BPMs will be useful for the future measurements in HL-LHC as they are placed close to the crab cavities, which also require good orbit and optics control. In the latest configuration, specifically designed for optics-based-calibration calculations, the value of the dispersion was not matched to zero in IR1 and IR5, and therefore it can be used as an alternative method for computing the BPM optics-based calibration factors.

Figures 6 and 7 show the designed $\beta$ function in horizontal and in vertical plane as well as the dispersion in the horizontal plane used in 2016 (top) and 2017 (bottom) in IR1 and IR5 for Beam 1 and Beam 2.

Ballistic optics measurements have been performed in three consecutive years: 2015, 2016, 2017. The optics configuration was not the same for all the years. In 2015, due to technical issues, measurements were only performed at injection energy $(450 \mathrm{GeV})$ [41]. Thanks to the promising results obtained in 2015, optics measurements were repeated in 2016 using the same ballistic configuration, this time at flattop energy $(6.5 \mathrm{TeV})$.

The main reason for measuring the BPM calibration factors in consecutive years was to evaluate the improvements performed in the BPMs during the yearly shutdown and to have the most recent value of the calibration factors commissioning. Several improvements were performed in the BPM electronics regarding minor software and several hardware problems during the extended end of year stop (2016-2017) [42].

Studies presented in this article are focused on the calibration factors measured at high-energy in 2016 and 2017 and their application to several different optics measurements during 2017 and 2018.

\section{A. Ballistic optics corrections}

During normal optics measurement procedure, $\beta$ function is measured several times for both beams. First, to
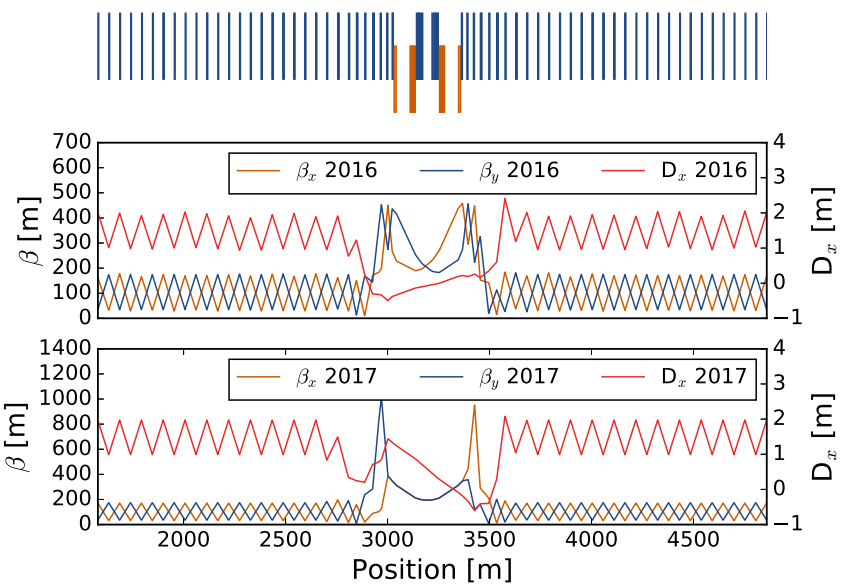

FIG. 7. Comparison of the horizontal model $\beta$, vertical model $\beta$ function and dispersion for the IR5: top 2016 and bottom 2017 (Beam 2).

compute local or global corrections and second, to validate the effectiveness of the corrections. Local optics corrections, based on the analysis of the phase advance propagation in the IR [12], are important to reduce $\beta$-beating in the IR in order to have the most accurate values of $\beta^{\phi}$ and therefore, accurate values of the calibration factors. If a large deviation is observed in the phase with respect to the model, i.e., phase-beating, the strength of the IR quadrupoles (in this case Q5, Q6, and Q7) are modified to compensate this phase advance deviation. In 2017, after the first optics measurements, local corrections were implemented in the machine by changing the strength of the quadrupoles Q5 and Q6 in the interaction region 1 with the main aim to decrease local errors of the IR1. Figures 8 and 9 show the phase advance measured in the IR1 before (blue) and after the corrections (orange) for Beam 1 and Beam 2, respectively.

Table II summarizes the local corrections performed in IR1. Their effect has been summarized in Tables II and III.

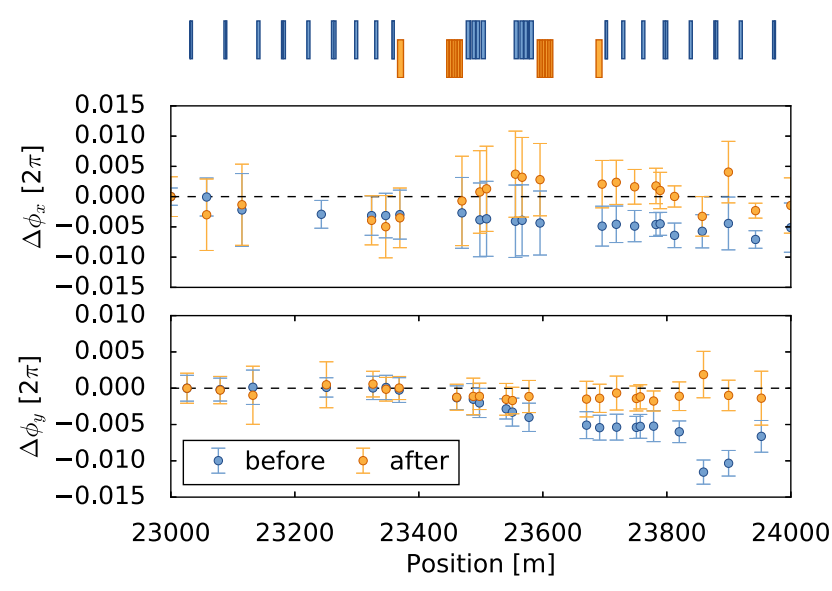

FIG. 8. Comparison of the phase $(\Delta \phi)$ in IR1 measured in 2017 for Beam 1: Horizontal plane (top) and vertical plane (bottom). 


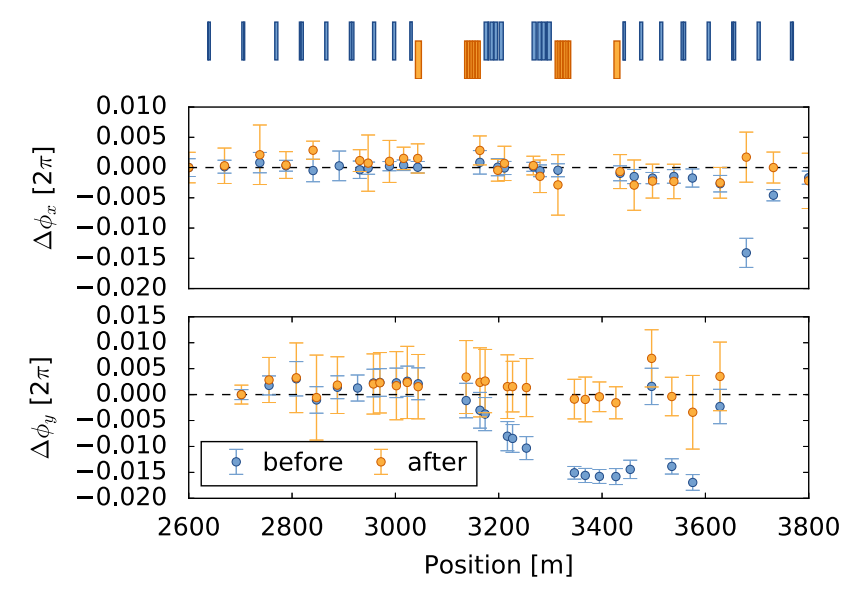

FIG. 9. Comparison of the phase $(\Delta \phi)$ in IR1 measured in 2017 for Beam 2: Horizontal plane (top) and vertical plane (bottom).

RMS $\beta$-beating in the interaction region was decreased in IR1 in both beams while $\beta$-beating in the arcs remained similar.

During the last set of measurements performed in 2017, the rms $\beta$-beating in the arcs was kept below $5 \%$ for both planes and beams.

\section{B. Ballistic optics measurements}

A comparison of the horizontal and vertical $\beta$ function obtained using $\beta^{\phi}$ and $\beta^{\mathrm{A}}$ approaches for IR1 and IR5 is shown in Figs. 10 and 11, respectively. A schematic of the IR lattice is placed in the top of both plots while measurements performed in 2016 and 2017 are shown in the top and bottom figures respectively. These comparisons are focused on the IR area in between the two Q4 (left and

TABLE II. Local corrections implemented in IR1.

\begin{tabular}{lccc}
\hline \hline Circuit & $\begin{array}{c}\Delta \mathrm{k} \\
\left(10^{-5} \mathrm{~m}^{-2}\right)\end{array}$ & $\begin{array}{c}\mathrm{k} \text { nominal } \\
\left(10^{-3} \mathrm{~m}^{-2}\right)\end{array}$ & $\begin{array}{c}\text { Correction } \\
(\%)\end{array}$ \\
\hline kq5.11b1 & -2.3 & -2.97 & 0.77 \\
kq5.11b2 & 3.4 & 3.8 & 0.90 \\
kq5.r1b1 & 3.1 & 3.1 & 1.0 \\
kq5.r1b2 & -2.5 & -3.6 & 0.70 \\
kq6.11b1 & 2.0 & 5.8 & 0.34 \\
kq6.r1b1 & -3.4 & -5.7 & 0.60 \\
kq6.r1b2 & 3.5 & 5.9 & 0.60 \\
\hline \hline
\end{tabular}

TABLE III. Rms $\beta$-beating before (b. corr.) and after (a. corr.) local corrections (2017).

\begin{tabular}{ccccccc}
\hline \hline & \multicolumn{2}{c}{ Beam 1 } & & \multicolumn{2}{c}{ Beam 2 } \\
\cline { 6 - 7 } & & Horizontal & Vertical & & Horizontal & Vertical \\
\hline \multirow{2}{*}{ rms $\frac{\Delta \beta}{\beta}(\%)$} & b. corr. & 4.1 & 4.3 & & 5.1 & 3.9 \\
& a. corr. & 4.3 & 3.5 & & 4.8 & 3.5 \\
\hline \hline
\end{tabular}

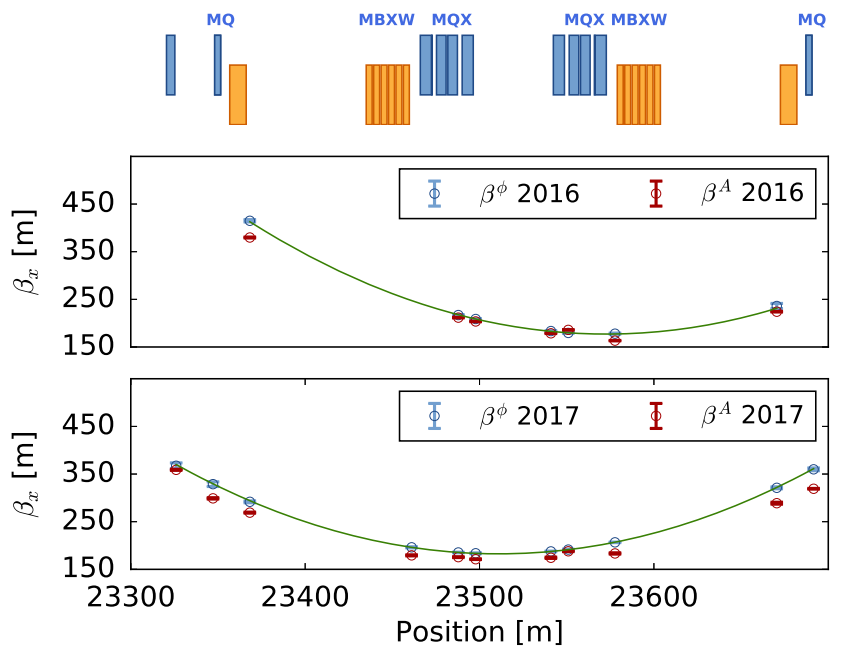

FIG. 10. Parabolic fit of $\beta^{\phi}$ measured in 2016 (top) and 2017 (bottom) in horizontal plane in IR1 Beam 1.

right), where the difference between the two $\beta$ approaches is more significant.

The green line plotted in Figs. 10 and 11 shows the parabolic fit to $\beta^{\phi}$ measurements in the IR 1 and 5 respectively. The parabolic behavior of the $\beta$ function could be affected by the b2 components due to magnetic imperfections of the MBXW dipoles. These errors have been simulated using the magnetic measurements via WISE [43], obtaining a deviation of a $0.005 \%$.

As can be seen in Figs. 10 and 11 the values given by the $\beta^{\mathrm{A}}$ approach are systematically lower than those given by $\beta^{\phi}$. The discrepancy between the two methods is attributed to a calibration error of the BPMs. The results for Beam 2 show the same behavior.

Figure 12 shows the measurement of the dispersion function in IR1 in 2017 using calibration-dependent approach $\left(D_{x}\right)$ and the calibration-independent method

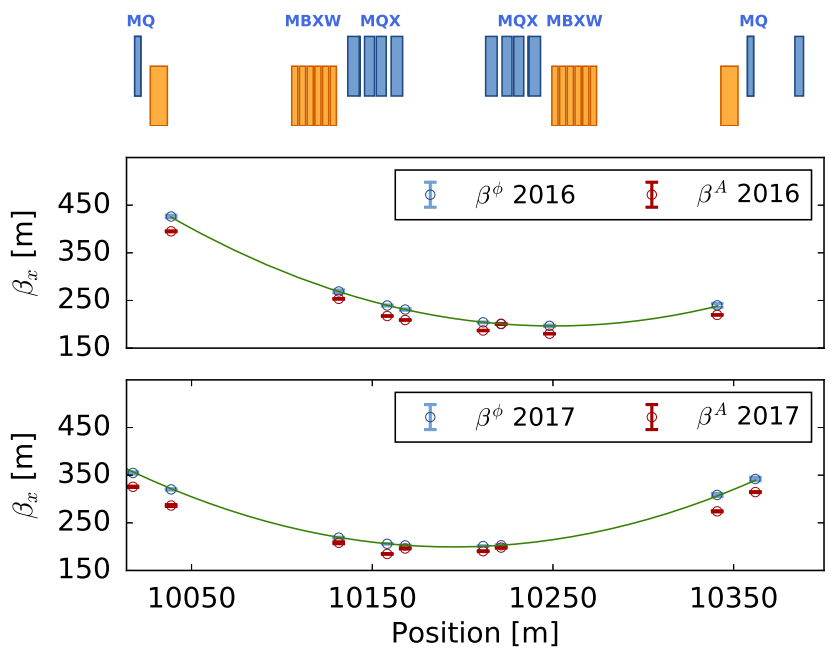

FIG. 11. Parabolic fit of $\beta^{\phi}$ measured in 2016 (top) and 2017 (bottom) in horizontal plane in IR5 Beam 1. 

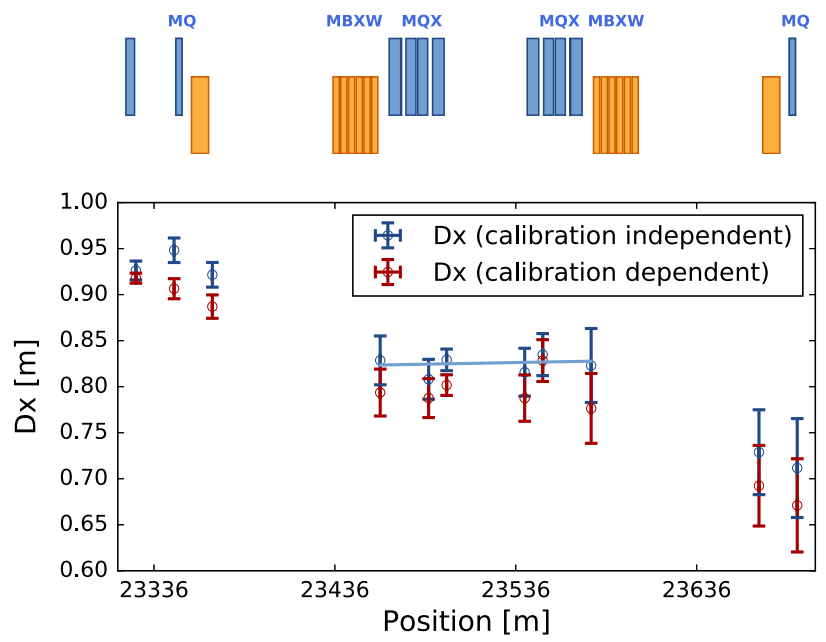

FIG. 12. Dispersion calculated in 2017 using calibrationdependent vs calibration-independent approach (IR1, Beam 1). Blue line represents the linear fit to the measured dispersion using calibration independent, $\mathrm{D}_{x}$.

$\left(D_{N, x} \sqrt{\beta^{\phi}}\right)$. (Red dots show the dispersion measurement biased by the calibration factors Eq. (26), while blue dots show the calculation of the dispersion function based on the normalized dispersion [Eq. (27)].) Dispersion functions have only been used for computing the calibration factors in Beam 1 since the dispersion function in Beam 2 is very small in IR1 and IR5. Values of dispersion close to 0 are currently measured with an associated error that fluctuates between $10 \%$ and $40 \%$.

Dispersion function in a drift depends linearly upon the longitudinal position. The results of a linear fit are illustrated in Figs. 12 and 13 corresponding to IR1 and IR5, respectively.
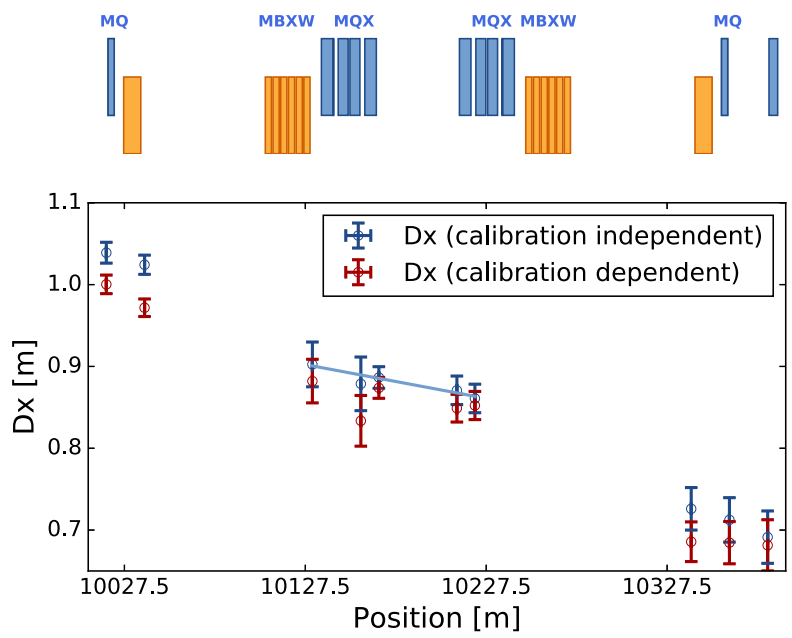

FIG. 13. Dispersion calculated in 2017 using calibrationdependent vs calibration-independent approach (IR5, Beam 1). Blue line represents the linear fit to the measured dispersion using calibration independent, $\mathrm{D}_{x}$.

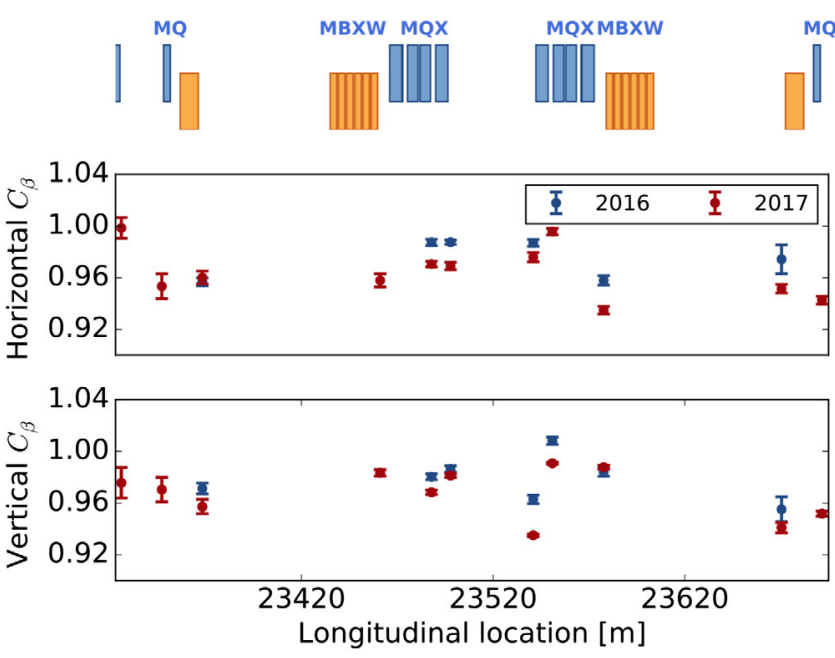

FIG. 14. Comparison of calibration factors measured at $6.5 \mathrm{TeV}$ in 2016 and 2017 (IR 1, Beam 1).

The error bars of the dispersion measurements are dominated by the statistical errors.

\section{Calibration factors 2016 vs 2017}

A comparison between the calibration factors calculated in 2016 and 2017 is shown in Figs. 14 and 15. It should be kept in mind that improvements were performed in the BPMs software and hardware between the measurements. Additionally, an histogram of the calibration factors and their uncertainties for both beams is shown Figs. 16 and 17.

\section{Calibration factors 2017: $\beta$ and dispersion}

Figure 18 compares the horizontal calibrations factors obtained using the two methods previously described. Each point represents the calibration factor measured at a given

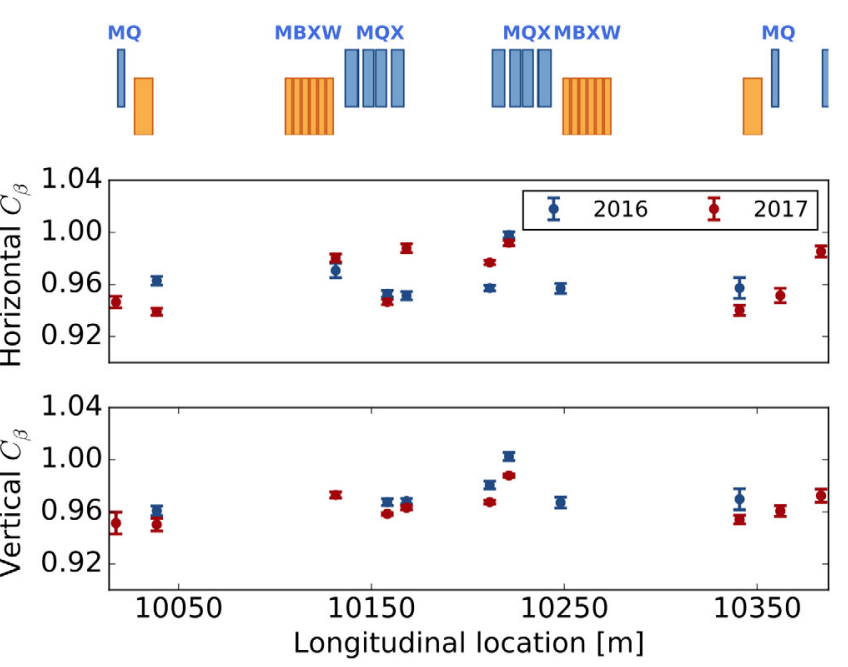

FIG. 15. Comparison of calibration factors measured at $6.5 \mathrm{TeV}$ in 2016 and 2017 (IR 5, Beam 1) for horizontal (top) and vertical (bottom) planes. 


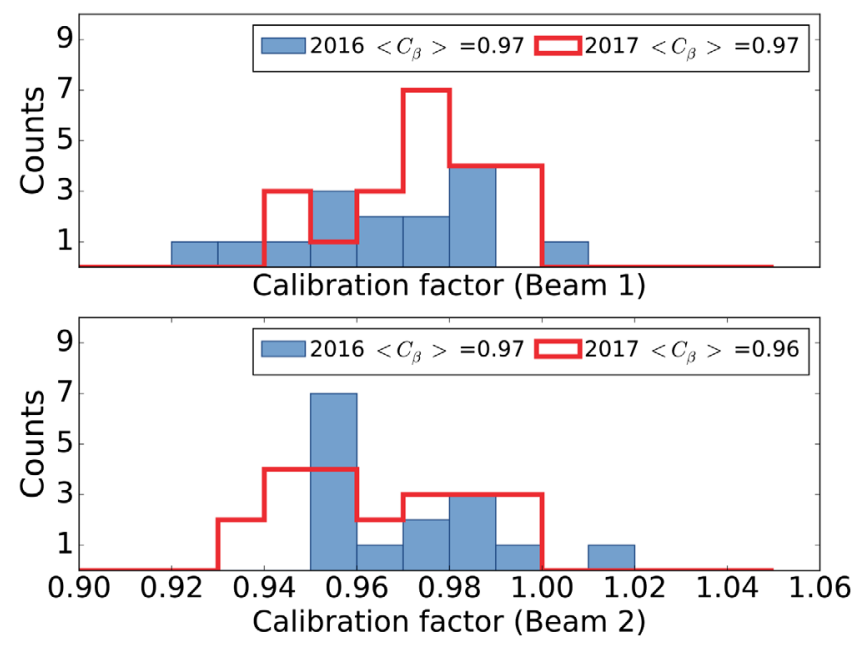

FIG. 16. Histogram of the calibration factor for Beam 1 and Beam 2 in 2016 and 2017 for horizontal and vertical planes.

BPM using the $\beta$ method and the dispersion approaches. A linear fit of the calibration factors $C_{\beta_{x}}$ as a function of $C_{D_{x}}$, shows that the values are compatible. $C_{D, x}$ accuracy fluctuates between $2 \%$ and $10 \%$ for both planes in Beam 1 while the accuracy of the calibration factors from amplitude, $C_{\beta, x}$, fluctuates between $0.3 \%$ and $1 \%$.

\section{E. Calibration factors 2017: Ballistic and averaging optics}

Calibration factors have also been computed using the ratio $\sqrt{\beta^{\mathrm{A}} / \beta^{\phi}}$ for different optics and then averaging over them. Their associated error bar have been computed as the standard deviation of the calibration factors over the optics measured. This study aims to justify the development of an
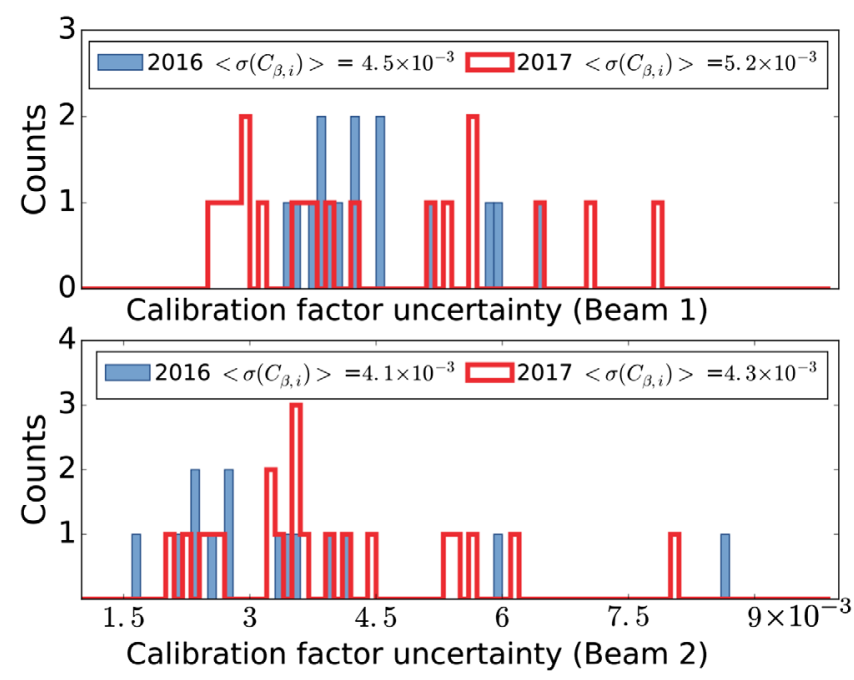

FIG. 17. Histogram of the calibration factor uncertainty for Beam 1 and Beam 2 in 2016 and 2017 for horizontal (top) and vertical (bottom) planes.

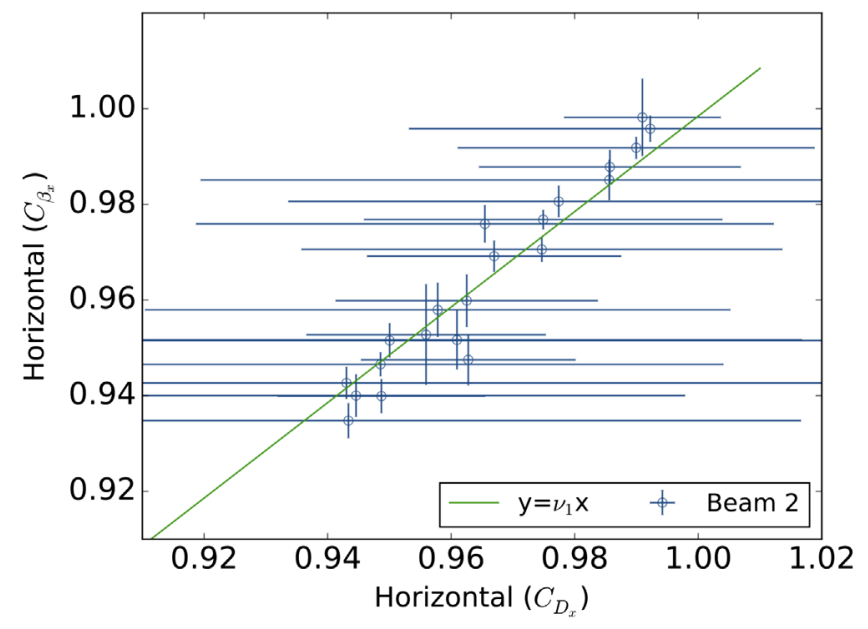

FIG. 18. Calibration factors using $\beta$ from amplitude vs calibration factors obtained using dispersion (Beam 1). The green line represent a first order polynomial fit of the data set- $C_{D_{x}}, C_{\beta_{x}}$, with a slope $\nu_{1}=0.998 \pm 0.02$.

optics configuration dedicated exclusively to the calibration factors calculation. It can be observed in Figs. 19 and 20 that the error bar obtained using a dedicated optics for the computation of the calibration factors is on average three times smaller than averaging the calibration factors over a set of optics.

\section{APPLICATIONS OF THE CALIBRATION FACTORS TO OTHER OPTICS}

Well calibrated BPMs could allow obtaining accurate $\beta^{*}$ measurements and, in general, the $\beta$ function in the

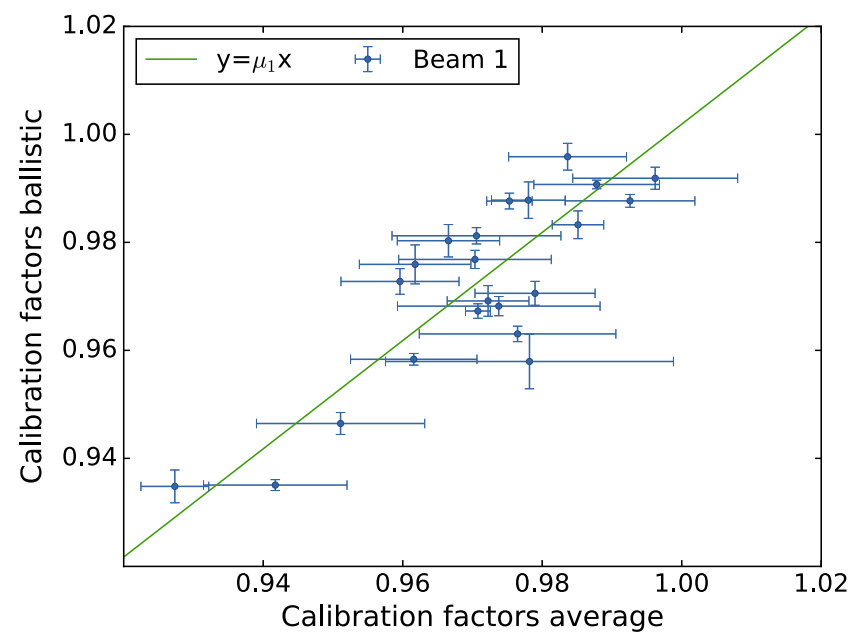

FIG. 19. Average calibration factor measured for different optics vs calibration factors measured using ballistic optics (horizontal and vertical planes, Beam 1). The green line represent a first order polynomial fit of the dataset-calibration factors ballistic, calibration factors average-with a slope $\mu_{1}=1.002 \pm 0.002$. 


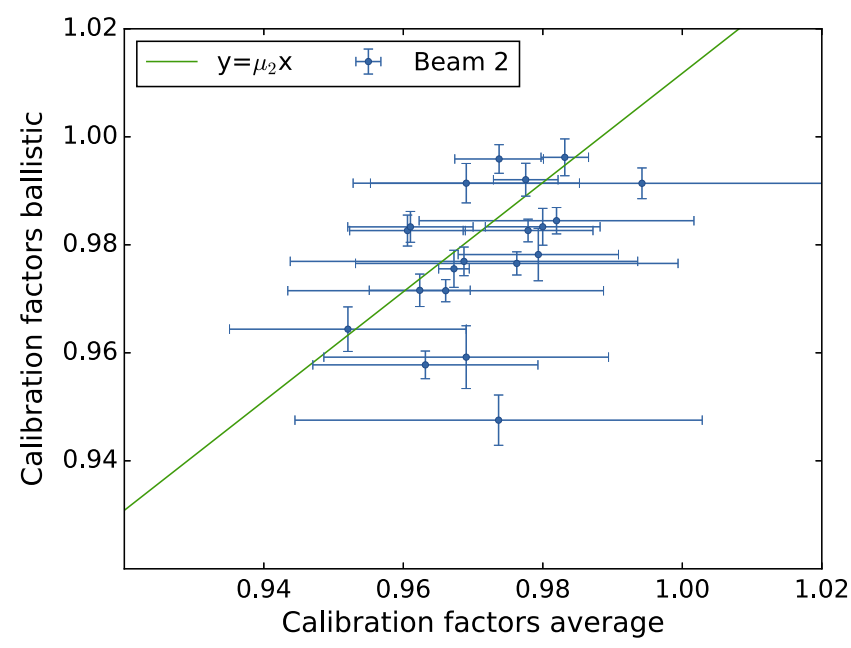

FIG. 20. Average calibration factor measured for different optics vs calibration factors measured using ballistic optics (horizontal and vertical planes, Beam 2). The green line shows the fit of the data set-calibration factors ballistic, calibration factors average - to a first-degree polynomial fit with slope $\mu_{2}=1.012 \pm 0.002$.

interaction regions directly from the amplitude of the Fourier spectra in the scenarios where the other methods present limitations.

This section summarizes a comparison of the values obtained using the $\beta$ from amplitude method before and after applying the calibration factors. Different optics configurations that have been used to validate the recalibration process are listed below, grouped according to the

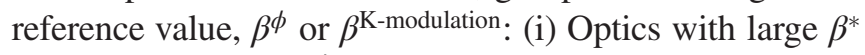
$\left(\beta^{*}>1 \mathrm{~m}\right)$ where $\beta^{\phi}$ can be used as a reference value.

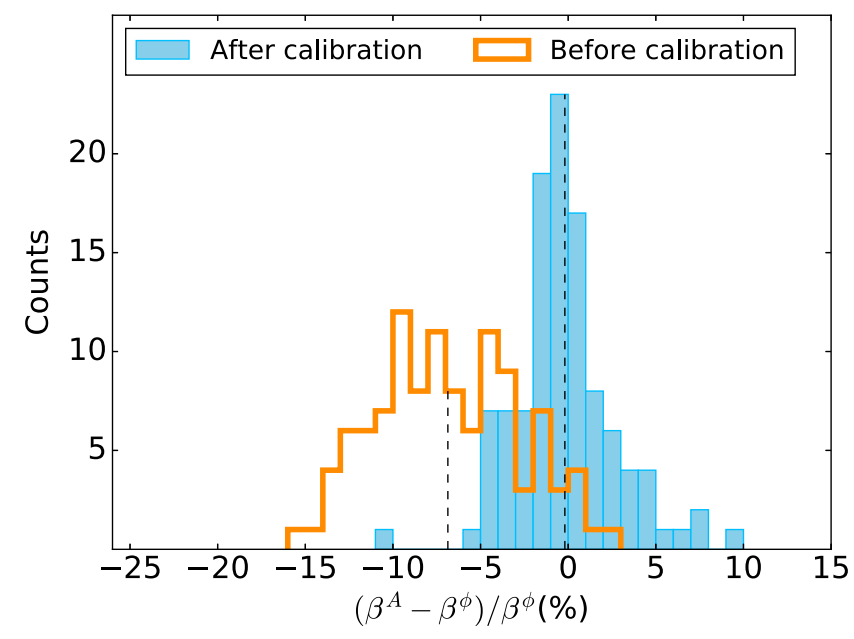

FIG. 21. Histogram of $\beta$-beating before and after calibration, using $\beta^{\phi}$ as reference in horizontal and vertical planes measured in several optics: Injection and flattop during 2017 and 2018 (Beam 1, horizontal and vertical planes, IR1 and IR5).

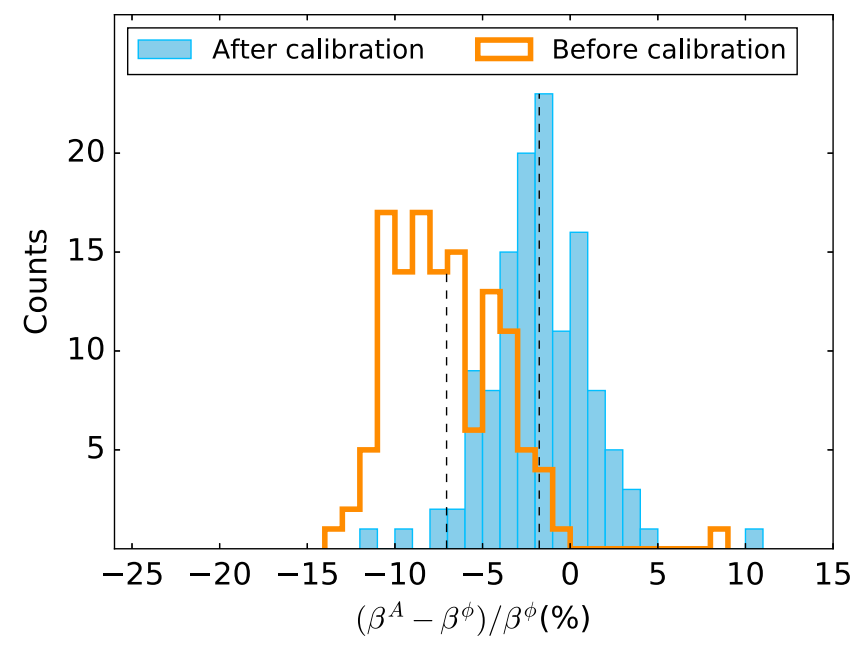

FIG. 22. Histogram of $\beta$-beating before and after calibration, using $\beta^{\phi}$ as reference in horizontal and vertical planes measured in several optics: Injection and flattop during 2017 and 2018 (Beam 2, horizontal and vertical planes, IR1 and IR5).

These optics are: flattop, injection and high- $\beta^{*}$ run. Figures 21 and 22 show a comparison of the $\beta^{\phi}$ and $\beta^{\mathrm{A}}$ beating measured at the stripline BPMs placed in the IRs 1 and 5, using those optics, before and after applying the calibration factors. The average $\left(\beta^{\mathrm{A}}-\beta^{\phi}\right) / \beta^{\phi}$ and its associated spread are shown in Table IV. From both Figs. 21 and 22 and Table IV it can be observed that, on average, the calibration errors have been compensated when recalibrating the instrumentation. The spread associated with the ratio $\left(\beta^{A}-\beta^{\phi}\right) / \beta^{\phi}$ is given by the combination of error bar associated to the $\beta^{\phi}$ and $\beta^{\mathrm{A}}$. (ii) Low $\beta^{*}$ runs where $\beta^{\mathrm{K} \text {-modulation }}$ can be used as a reference value. Several runs of low $\beta^{*}$ have been performed in the last two years: $\beta^{*}=30 \mathrm{~cm}$ and $\beta^{*}=40 \mathrm{~cm}$ in 2017 and $\beta^{*}=$ $25 \mathrm{~cm}$ and $\beta^{*}=30 \mathrm{~cm}$ in $2018[44,45]$. Figures 23 and 24 show a comparison of the $\beta$-beating of the $\beta^{\mathrm{K} \text {-modulation }}$ and $\beta^{\mathrm{A}}$ measured at the stripline BPMs placed in the IRs 1 and 5 before and after compensated the calibration factors. Average $\left(\beta^{\mathrm{A}}-\beta^{\mathrm{K} \text {-modulation }}\right) / \beta^{\mathrm{K} \text {-modulation }}$ are shown Table $\mathrm{V}$ and its associated spread. A shift in the average $\beta$ beating has also been observed after compensating the calibration factors. Calibration factors have been

TABLE IV. Average and standard deviation of the distributions $\left(\beta^{\mathrm{A}}-\beta^{\phi}\right) / \beta^{\phi}$ before and after applying the calibration factors.

\begin{tabular}{|c|c|c|c|c|}
\hline & \multicolumn{2}{|c|}{ Not calibrated } & \multicolumn{2}{|c|}{ Calibrated } \\
\hline & Beam 1 & Beam 2 & Beam 1 & Beam 2 \\
\hline$\overline{\left(\beta^{\mathrm{A}}-\beta^{\phi}\right) / \beta^{\phi}}(\%)$ & -6.9 & -7.1 & -0.2 & -1.8 \\
\hline$\sigma\left(\beta^{\mathrm{A}}-\beta^{\phi}\right) / \beta^{\phi}(\%)$ & 4.0 & 3.1 & 2.9 & 2.9 \\
\hline
\end{tabular}




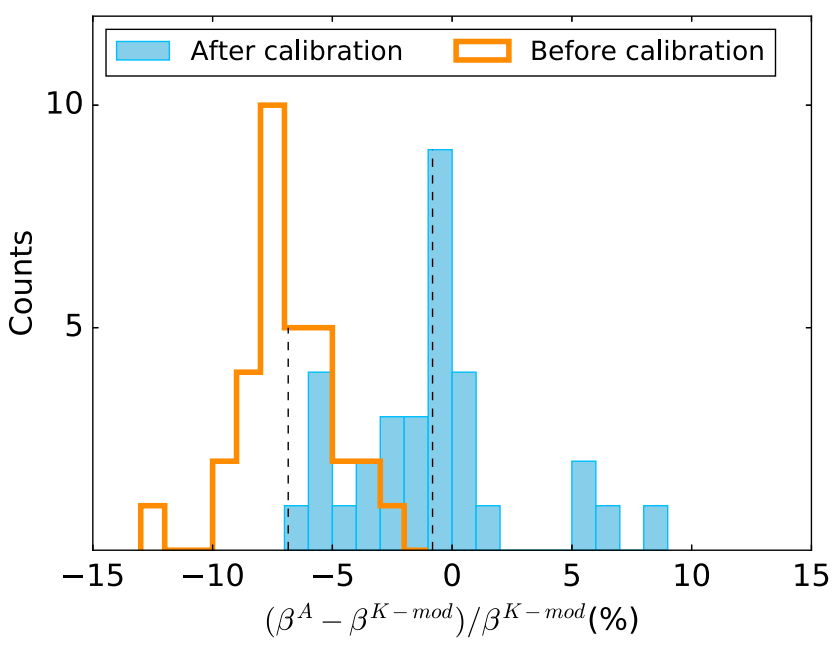

FIG. 23. Histogram of $\beta$-beating before and after calibration using $\beta^{\mathrm{K} \text {-modulation }}$ as reference values (Beam 1).

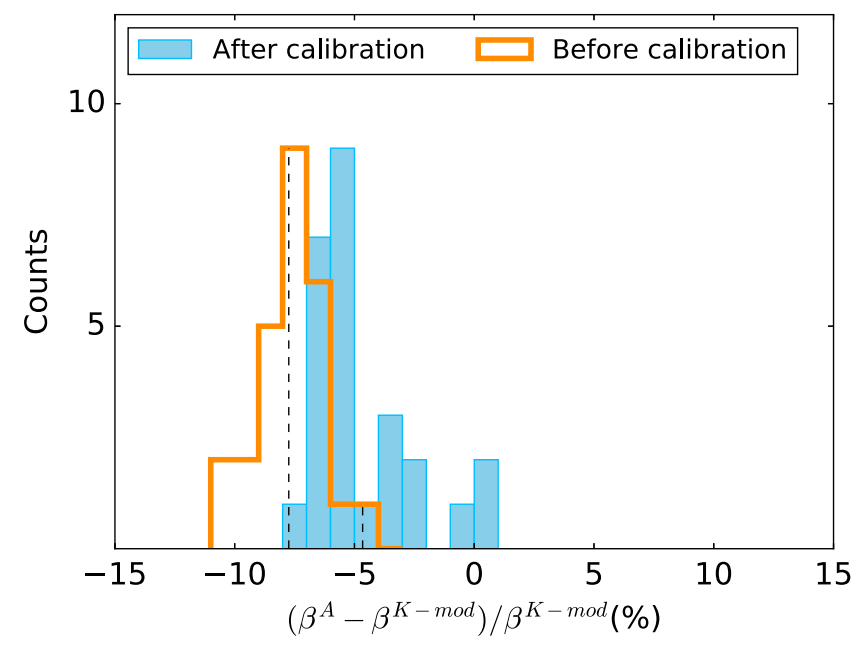

FIG. 24. Histogram of $\beta$-beating before and after calibration using $\beta^{\mathrm{K}-\text { modulation }}$ as reference values (Beam 2).

successfully implemented in Beam 1 where the average $\beta$-beating between the two approaches is $-0.9 \%$. Nonetheless, a remaining $-4.7 \%$ is observed in Beam 2 and will require further investigation.

TABLE V. Average and standard deviation of the distributions $\left(\beta^{\mathrm{A}}-\beta^{\mathrm{K}-\mathrm{mod}}\right) / \beta^{\mathrm{K}-\bmod }$ and $\left(\beta^{A, \mathrm{cal}}-\beta^{\mathrm{K}-\bmod }\right) / \beta^{\mathrm{K}-\bmod }$

\begin{tabular}{cccccc}
\hline \hline & \multicolumn{2}{c}{ Not calibrated } & & \multicolumn{2}{c}{ Calibrated } \\
\cline { 2 - 3 } \cline { 5 - 6 } & Beam 1 & Beam 2 & & Beam 1 & Beam 2 \\
\hline$\overline{\left(\beta^{\mathrm{A}, \text { cal }}-\beta^{\mathrm{K}-m o d}\right) /}$ & -6.8 & -7.8 & & -0.9 & -4.7 \\
$\beta^{\mathrm{K}-\text { mod }}(\%)$ & & & & \\
$\begin{array}{c}\sigma\left(\beta^{\mathrm{A}, \mathrm{cal}}-\beta^{\mathrm{K}-\mathrm{mod}}\right) / \\
\beta^{\mathrm{K}-\text { mod }}(\%)\end{array}$ & 1.9 & 1.4 & & 3.5 & 2.2 \\
\hline \hline
\end{tabular}

\section{CONCLUSIONS}

BPM calibration factors have been computed for the first time in LHC using measured optics functions. This method, denoted as optics-measurement-based BPM calibration, is based on the analysis of two different optics functions: $\beta$ function and dispersion. A dedicated optics configuration, known as ballistic optics, has been developed for these studies. A drift space is generated in the vicinity of the IP, allowing us to measure the $\beta$-function using the phase approach with a precision of about $0.5 \%$. The achieved precision on $\beta$-function has allowed computing the BPM calibration factors by comparing $\beta^{\mathrm{A}}$ to $\beta^{\phi}$ with an average uncertainty in the subpercent level. The promising results obtained in 2016, in terms of BPM accuracy and uncertainty achieved, motivated the further development of the ballistic configuration. In 2017, the drift space was extended by switching off the Q4 quadrupoles and, at the same time, by not matching the dispersion function to zero in IR1 and IR5. These two developments allowed us to increase the range of BPMs being calibrated and to incorporate dispersion function in the optics-measurement-based calibration approach.

The comparison between calibration factors measured using ballistic and the ones obtained averaging different optics, proves that the $\beta^{\phi}$ precision is the main limitation in the calibration-factor calculation. Nonetheless, currently the possible dependency of the calibration factors over time is not included in the ballistic error bar. This dependency will be addressed in LHC Run 3 and in view of HL-LHC.

Several optics configurations have been analyzed in order to estimate the impact of BPM recalibration on the $\beta^{\mathrm{A}}$ approach. Those optics are characterized by the $\beta$ function accuracy in the stripline BPMs placed in IR1 and IR5; measured either using $\beta^{\phi}$ or $\beta^{\mathrm{K}-\text { modulation }}$ approach. It has been observed that the $\beta$-beating with respect to the phase, $\left(\beta^{\mathrm{A}}-\beta^{\phi}\right) / \beta^{\mathrm{A}}$, is reduced on average a $6 \%$ after the BPM recalibration. If $\beta^{\mathrm{K} \text {-modulation }}$ is used as a reference value, the measured $\beta$-beating $\left(\beta^{\mathrm{A}}-\right.$ $\left.\beta^{\mathrm{K} \text {-modulation }}\right) / \beta^{\mathrm{K} \text {-modulation }}$ decreases on average a $6 \%$ for Beam 1, and a $3 \%$ for Beam 2 .

The large error-bars associated with the dispersion measurements - in contrast to the subpercent error bars associated to the $\beta$-function-propagate directly to the calibration factor calculations affecting the accuracy of the measurements. The agreement between the calibration factors obtained using $\beta$-function has been used as a validation for the optics-measurement-based calibration approach. A more in-depth study of the dispersion error bar should be further performed before recalibrating the BPMs using this technique.

\section{ACKNOWLEDGMENTS}

Particular thanks go to Gianluigi Arduini and Massimo Giovannozzi for proofreading the manuscript, and the 
Optics Measurements and Corrections team for providing useful and valuable insights.

\section{APPENDIX A: $\beta$-BEATING EVOLUTION IN A LATTICE WITH ERRORS}

The perturbed $\beta$ function in a ring is expressed as a function of the amplitude and phase of the generating driving term $f_{2000}$ and the unperturbed $\beta_{\text {model }}$ function, as

$$
\beta=\beta^{\text {model }}\left(1+32\left|f_{2000}\right|^{2}+8\left|f_{2000}\right| \sin q_{2000}\right),
$$

where $\left|f_{2000}\right|$ and $q_{2000}$ are the amplitude and phase of the generating function term. The relationship between $f_{2000}$ and gradient errors $\delta K_{w}$ is given by [46],

$$
f_{2000, j}=\frac{\sum_{w}^{W} \beta_{x, w}^{\text {model }} \delta K_{w} e^{2 i \Delta \phi_{x, w j}^{\text {model }}}}{8\left(1-e^{4 \pi i Q_{x}}\right)} .
$$

With many small random errors $\left|f_{2000}\right|$ and $\sin q_{2000}$ would tend to be uncorrelated giving a ring-average $\beta$-beating of

$$
\left\langle\frac{\Delta \beta_{i}}{\beta_{i}^{\text {model }}}\right\rangle=32\left\langle\left|f_{2000}\right|^{2}\right\rangle
$$

The standard deviation, $\sigma$, of the $\beta$-beating around the ring is given by

$$
\sigma\left(\frac{\Delta \beta_{i}^{\phi}}{\beta^{\text {model }}}\right)=\sqrt{\frac{1}{C} \int_{0}^{C} 64\left|f_{2000}\right|^{2} \sin ^{2} q_{2000} d s .}
$$

Using that $\sin ^{2} x=(1-\cos 2 x) / 2$ and, again, the assumption that $\left|f_{2000}\right|$ and $q_{2000}$ are uncorrelated, the standard deviation takes the form

$$
\sigma\left(\frac{\Delta \beta_{i}^{\phi}}{\beta_{i}^{\text {model }}}\right)=\sqrt{32\left\langle\left|f_{2000}\right|^{2}\right\rangle}
$$

From Eqs. (A3) and (A5) the following identity is obtained

$$
\left\langle\frac{\Delta \beta_{i}^{\phi}}{\beta_{i}^{\text {model }}}\right\rangle=\sigma^{2}\left(\frac{\Delta \beta_{i}^{\phi}}{\beta_{i}^{\text {model }}}\right)
$$

which implies that the ring-average $\beta$ function increases with the square of the standard deviation of the $\beta$-beating,
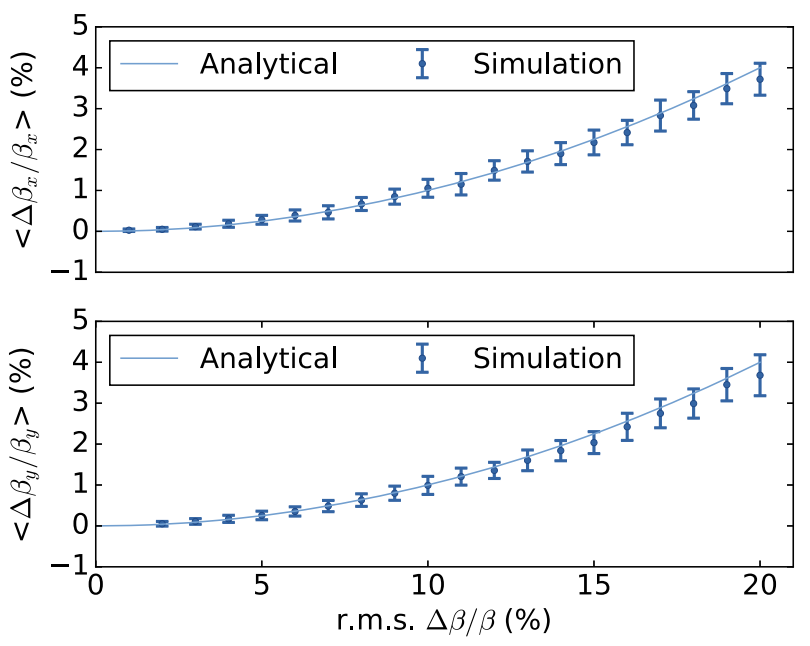

FIG. 25. Beta-beating average as a function of the beta-beating rms considering only BPMs placed in the ARCs.

also known as rms $\beta$-beating. This seems to be a universal property of all lattices since no assumptions on the lattice is made other than random error sources. The relation between these two quantities, average $\beta$-beating and rms $\beta$-beating have been studied in MADX simulations. Figure 25 shows the LHC ballistic simulations including the prediction from Eq. (A6).

\section{APPENDIX B: TUNE UNCERTAINTY PROPAGATION IN $\beta^{\mathrm{A}}$-CALCULATION}

The effect of the natural and driven tune uncertainty in the ac-dipole compensation have been analytically studied. Performing error propagation in Eq. (10) Assuming that the natural and the driven tune uncertainties are equal, it is possible to obtain the error introduced in the $\beta_{i}$ function: Assuming that the uncertainty of the natural tune $\sigma_{Q}$ and the driven tune $\sigma_{Q_{d}}$ are equivalent and equal to $\sigma_{Q}$

Lambda factor, used in the compensation of the acdipole effect is given by:

$$
\lambda=\frac{\sin \left[\pi\left(\mathrm{Q}_{\mathrm{d}}-\mathrm{Q}\right)\right]}{\left.\sin \left[\pi\left(\mathrm{Q}_{\mathrm{d}}+\mathrm{Q}\right)\right)\right]}
$$

$\Delta \lambda^{2}=\left(\frac{\partial \lambda}{\partial Q_{d}} \sigma\left(Q_{d}\right)\right)^{2}+\left(\frac{\partial \lambda}{\partial Q} \sigma(Q)\right)^{2} \approx\left(\frac{\partial \lambda}{\partial Q} \sigma(Q)\right)^{2}$

Assuming $\sigma(Q) \approx \sigma\left(Q_{d}\right)$ and using the parity properties of the sinusoidal functions, the two terms of Eq. (B1) are equivalent. Applying trigonometrical identities, the numerator of Eq. (B1) can be simplified, 


$$
\begin{aligned}
\Delta \lambda^{2}= & 2\left(\frac{\pi \cos \left[\pi\left(\mathrm{Q}_{\mathrm{d}}-\mathrm{Q}\right)\right] \sin \left[\pi\left(\mathrm{Q}_{\mathrm{d}}+\mathrm{Q}\right)\right]-\pi \cos \left[\pi\left(\mathrm{Q}_{\mathrm{d}}+\mathrm{Q}\right)\right] \sin \left[\pi\left(\mathrm{Q}_{\mathrm{d}}-\mathrm{Q}\right)\right]}{\sin ^{2} \pi\left(Q_{d}+Q\right)}\right)^{2} \sigma_{Q}^{2} \\
= & 2\left(\frac{\pi\left(\cos \pi Q_{d} \cos \pi Q+\sin \pi Q_{d} \sin \pi Q\right) \times\left(\sin \pi Q_{d} \cos \pi Q+\cos \pi Q_{d} \sin \pi Q\right)}{\sin ^{2} \pi\left(Q_{d}+Q\right)}\right)^{2} \sigma_{Q}^{2} \\
& +2\left(\frac{\pi\left(\sin \pi Q_{d} \sin \pi Q-\cos \pi Q_{d} \cos \pi Q\right) \times\left(\sin \pi Q \cos \pi Q_{d}-\sin \pi Q_{d} \cos \pi Q_{d}\right)}{\sin ^{2} \pi\left(Q_{d}+Q\right)}\right)^{2} \sigma_{Q}^{2} \\
= & 2 \pi^{2}\left(\frac{2 \cos \pi Q_{d} \sin \pi Q_{d}\left(\cos ^{2} \pi Q+\sin ^{2} \pi Q\right)}{\sin ^{2} \pi\left(Q_{d}+Q\right)}\right)^{2} \sigma_{Q}^{2}=2 \pi^{2}\left(\frac{2 \cos \pi Q_{d} \sin \pi Q_{d}}{\sin ^{2} \pi\left(Q_{d}+Q\right)}\right)^{2} \sigma_{Q}^{2}
\end{aligned}
$$

The maximum uncertainty introduced in the $\beta$ calculation due to previously computed error in $\lambda$ calculation is given by:

$$
\frac{\partial \beta_{i}}{\partial \lambda}=\frac{\left(A_{i}^{\mathrm{D}, \text { meas }}\right)^{2}}{2 J_{C}^{\mathrm{D}}} \frac{2}{(1-\lambda)^{2}}
$$

[1] F. Zimmermann, S. Fartoukh, and R. W. Assmann, Measuring beta functions and dispersion in the early LHC, in Proceedings of 8th European Particle Accelerator Conference, Paris, France (European Physical Society, Geneva, 2002).

[2] Large Hadron Collider (LHC) Technical Design Report v. 1: the LHC Main Ring”. https://cds.cern.ch/record/782076/ files/CERN-2004-003-V1.pdf.

[3] Large Hadron Collider (LHC) Technical Design Report v. 2: the LHC Infrastructure and General Services." http://cds .cern.ch/record/815187/files/CERN-2004-003-V2.pdf.

[4] Large Hadron Collider (LHC) Technical Design Report v. 1: the LHC Injector Chain." http://cds.cern.ch/record/ 823808/files/CERN-2004-003-V3.pdf.

[5] High-Luminosity Large Hadron Collider (HL-LHC) Technical Design Report V. 0.1 https://e-publishing.cern.ch/ index.php/CYRM/issue/view/40/showToc.

[6] D. Amorim et al., High-energy LHC design, in Proceedings of 9th International Particle Accelerator Conference, Vancouver, BC, Canada (JACoW, Geneva, 2017).

[7] M. Benedikt et al., FCC: Colliders at the energy frontier, in Proceedings of 9th International Particle Accelerator Conference, Vancouver, BC, Canada (JACoW, Geneva, 2017).

[8] A. Seryi et al., Overview of design development of FCC-hh experimental interaction regions, in Proceedings of 8th International Particle Accelerator Conference, Copenhagen, Denmark (JACoW, Geneva, 2018).

[9] CERN Document Server: ATLAS, http://cds.cern.ch/ collection/ATLAS? In.

[10] CERN Document Server: CMS, http://cds.cern.ch/ collection/CMS?ln.
[11] R. Tomás et al., Record low $\beta$ - beating in the LHC, Phys. Rev. Accel. Beams 15, 091001 (2012).

[12] J. M. C. de Portugal, F. Carlier, A. Garcia-Tabares, A. Langner, E. H. Maclean, L. Malina, T. Persson, P. Skowronski, and R. Tomás, Local Optics Corrections in the HL-LHC IR, in Proceedings of 7th International Particle Accelerator Conference, Busan, Korea (JACoW, Geneva, 2016).

[13] A. Langner, J. C. de Portugal, P. Skowronski, and R. Tomás, Developments of the segment-by-segment technique for optics corrections in the LHC, in Proceedings of 6th International Particle Accelerator Conference, Richmond VA, USA (JACoW, Geneva, 2015).

[14] J. C. de Portugal, F. Carlier, A. Langner, T. Persson, P. Skowronski, and R. Tomás, OMC software improvements in 2014, in Proceedings of 6th International Particle Accelerator Conference, Richmond VA, USA (JACoW, Geneva, 2015).

[15] F. Carlier and R.Tomás, Accuracy and feasibility of the $\beta^{*}$ measurements for LHC and high luminosity using Kmodulation, Phys. Rev. Accel. Beams 20, 011005 (2017).

[16] P. Thrane, Measuring $\beta^{*}$ in SuperKEKB with K Modulation, Report No. CERN-THESIS-2018-300, 2018, https:// cds.cern.ch/record/2652855.

[17] M. Hofer et al., K-Modulation for future High energy colliders, in Proceedings of 10th International Particle Accelerator Conference, Melbourne, Australia (JACoW, Geneva, Switzerland, 2019).

[18] M. Kuhn, V. Kain, A. Langner, and R. Tomas, First KModulation measurements in the LHC during run 2, in Proceedings of IBIC2015, Melbourne, Australia (JACoW, Geneva, 2016).

[19] P. Thrane, R. Tomás, A. Koval, K. Ohmi, Y. Ohnishi, and A. Wegscheider, Measuring $\beta^{*}$ in SuperKEKB with $\mathrm{K}$ Modulation, Phys. Rev. Accel. Beams 23, 012803, January 2020.

[20] P. Castro, Luminosity and beta function measurement at the electron-positron collider ring $\mathrm{LEP}, \mathrm{PhD}$ thesis, Valencia University, 1996.

[21] A. Wegscheider, A. Franchi, A. Langner, and R. Tomás, Analytical $\mathrm{N}$ beam position monitor method, Phys. Rev. Accel. Beams 20, 111002 (2017).

[22] A. Langner and R. Tomás, Optics measurement algorithms and error analysis for the proton energy frontier, Phys. Rev. Accel. Beams 18, 031002 (2015). 
[23] A. Langner, G. Benedetti, M. Carlà, U. Iriso, Z. Martí, J. C. de Portugal, and R. Tomás, Utilizing the $N$ beam position monitor method for turn-by-turn optics measurements, Phys. Rev. Accel. Beams 19, 092803 (2016).

[24] L. Malina, J. C. de Portugal, T. Persson, P.Skowroński, R. Tomás, A. Franchi, and S. Liuzzo, Improving the precision of linear optics measurements based on turn-by-turn beam position monitor data after a pulsed excitation in lepton storage rings, Phys. Rev. Accel. Beams 20, 082802 (2017).

[25] X. Shen, S. Y. Lee, M. Bai, S. White, G. RobertDemolaize, Y. Luo, A. Marusic, and R. Tomas, Application of independent component analysis to ac dipole based optics measurement and correction at the Relativistic Heavy Ion Collider, Phys. Rev. Accel. Beams 16, 111001 (2013).

[26] R. J. Steinhagen, A. Boccardi, E. Calvo Giraldo, M. Gasior, J. L. Gonzalez, and O. R. Jones, On the continuous measurement of the LHC $\beta$-function-prototype studies at the SPS, in Proceedings of PAC09, Vancouver, BC, Canada (TRIUMF, Vancouver, 2010), p. 5076.

[27] F. Carlier et al., LHC optics measurment and correction software progress and plans, in Proceedings of 9th International Particle Accelerator Conference, Vancouver, BC, Canada (JACoW, Geneva, 2018).

[28] P. Castro et al., Betatron fucntion measruement at LEP using the BOM 1000 turns facility, https://cds.cern.ch/ record/250451/files/CM-P00061230.pdf.

[29] M. Wendt, LHC BPMs status quo and introduction in the WP2/WP13 meeting on LHC BPM system requirements, https://indico.cern.ch/event/716801/.

[30] R. Calaga, R. Tomás, and F. Zimmermann, BPM calibration independent LHC optics correction, in Proceedings of PAC07, Albuquerque, New Mexico, USA https://accelconf .web.cern.ch/accelconf/p07/PAPERS/THPAS091.PDF.

[31] A. Verdier, Alignment optics for LHC, LHC Project Note 325, October 6, 2003.

[32] L. Van Riesen-Haupt, Advanced Accelerator Interaction Region Optics for LHC Operation and Future Hadron Colliders, PhD. thesis, University of Oxford, 2019.

[33] J. P. Koutchouk, Measurement of the beam position in the LHC main rings, Report No. LHC-BPM-ES-0004, 2002, https://cds.cern.ch/record/1068133.

[34] A. G.-T. Valdivieso, F. Carlier, J. C. de Portugal, C. A. G. Bonilla, A. Langner, E. H. Maclean, L. Malina, M. J. McAteer, T. H. B. Persson, P. Skowronski, and R. Tomás,
LHC injection optics measurements at commissioning, Report No. CERN-ACC-NOTE-2016-0033, 2015.

[35] T. Persson, F. Carlier, J. C. de Portugal, A. G.-T. Valdivieso, A. Langner, E. H. Maclean, L. Malina, P. Skowronski, B. Salvant, and R. Tomás, A. C. García Bonilla, LHC optics commissioning: A journey towards 1\% optics control, Phys. Rev. Accel. Beams 20, 061002 (2017).

[36] D. W. Wolf, Master thesis, Analysis of tune modulations in the LHC, CERN-THESIS-2018-251, https://cds.cern.ch/ record/2647994?.

[37] R. Miyamoto, S. Kopp, A. Jansson, and M. J. Syphers, Parametrization of the driven betatron oscillation, Phys. Rev. Accel. Beams, 11, 084002 (2008).

[38] L. Malina et al., Performance Optimisation of Turn-byTurn Beam Position Monitor Data Harmonic Analysis, in Proceedings of 9th International Particle Accelerator Conference, Vancouver, BC, Canada, http://dx.doi.org/ 10.18429/JACoW-IPAC2018-THPAF045.

[39] Methodical accelerator design, MADX, http://madx.web .cern.ch/madx/.

[40] R. J. Steinhagen, Tune and chromaticity diagnostics, CERN Report No. CERN-2009-005, https://cds.cern.ch/ record/1213281/files/p317.pdf.

[41] J. Coello, A. García-Tabarés, L. Malina, B. Salvachua, P. Skowronski, M. Solfaroli, R. Tomás, and J. Wenninger, MD Test of a Ballistic Optics, Report No. CERN-ACCNote-2016-0008, 2016.

[42] E. Bravin and J. Wenninger, BI changes in LBOC meeting, Tuesday 25 Apr 2017, https://indico.cern.ch/event/632455/ contributions/2557667/attachments/1449020/2233732/ LBOC.Systems.JW.25April17.pdf.

[43] P. Hagen et al., WISE: A Simulation of the LHC Optics including Magnet Geometrical Data, LHC-PROJECT-Report-1123, CERN-LHC-PROJECT-Report-1123, https:// cds.cern.ch/record/1123714.

[44] S. Fartoukh, Achromatic telescopic squeezing, Phys. Rev. Accel. Beams, 16, 111002 (2013).

[45] S. Fartoukh et al., Experimental validation of the Achromatic Telescopic Squeezing (ATS) scheme at the LHC, 2017, J. Phys. Conf. Ser. 874, 012010 (2017).

[46] A. Franchi, Error analysis of linear optics measurements via turn-by-turn beam position data in circular accelerators, arXiv:1603.00281v5, https://arxiv.org/pdf/1603.00281.pdf A. Franchi ESRF, Grenoble, France (Dated: April 18, 2018). 